\title{
Mass loss rates of a sample of irregular and semiregular M-type AGB-variables
}

\author{
H. Olofsson ${ }^{1}$, D. González Delgado ${ }^{1}$, F. Kerschbaum ${ }^{2}$, and F. L. Schöier ${ }^{3}$ \\ 1 Stockholm Observatory, SCFAB, 10691 Stockholm, Sweden \\ 2 Institut für Astronomie, Türkenschanzstrasse 17, 1180 Wien, Austria \\ ${ }^{3}$ Leiden Observatory, PO Box 9513, 2300 RA Leiden, The Netherlands
}

Received 27 March 2002 / Accepted 4 June 2002

\begin{abstract}
We have determined mass loss rates and gas expansion velocities for a sample of 69 M-type irregular (IRV; 22 objects) and semiregular (SRV; 47 objects) AGB-variables using a radiative transfer code to model their circumstellar CO radio line emission. We believe that this sample is representative for the mass losing stars of this type. The (molecular hydrogen) mass loss rate distribution has a median value of $2.0 \times 10^{-7} M_{\odot} \mathrm{yr}^{-1}$, and a minimum of $2.0 \times 10^{-8} M_{\odot} \mathrm{yr}^{-1}$ and a maximum of $8 \times 10^{-7} M_{\odot} \mathrm{yr}^{-1}$. M-type IRVs and SRVs with a mass loss rate in excess of $5 \times 10^{-7} M_{\odot} \mathrm{yr}^{-1}$ must be very rare, and among these mass losing stars the number of sources with mass loss rates below a few $10^{-8} M_{\odot} \mathrm{yr}^{-1}$ must be small. We find no significant difference between the IRVs and the SRVs in terms of their mass loss characteristics. Among the SRVs the mass loss rate shows no dependence on the period. Likewise the mass loss rate shows no correlation with the stellar temperature. The gas expansion velocity distribution has a median of $7.0 \mathrm{~km} \mathrm{~s}^{-1}$, and a minimum of $2.2 \mathrm{~km} \mathrm{~s}^{-1}$ and a maximum of $14.4 \mathrm{~km} \mathrm{~s}^{-1}$. No doubt, these objects sample the low gas expansion velocity end of AGB winds. The fraction of objects with low gas expansion velocities is very high, about $30 \%$ have velocities lower than $5 \mathrm{~km} \mathrm{~s}^{-1}$, and there are objects with velocities lower than $3 \mathrm{~km} \mathrm{~s}^{-1}$ : V584 Aql, T Ari, BI Car, RX Lac, and $\mathrm{L}^{2}$ Pup. The mass loss rate and the gas expansion velocity correlate well, a result in line with theoretical predictions for an optically thin, dust-driven wind. In general, the model produces line profiles which acceptably fit the observed ones. An exceptional case is R Dor, where the high-quality, observed line profiles are essentially flat-topped, while the model ones are sharply double-peaked. The sample contains four sources with distinctly double-component CO line profiles, i.e., a narrow feature centered on a broader feature: EP Aqr, RV Boo, X Her, and SV Psc. We have modelled the two components separately for each star and derive mass loss rates and gas expansion velocities. We have compared the results of this M-star sample with a similar C-star sample analysed in the same way. The mass loss rate characteristics are very similar for the two samples. On the contrary, the gas expansion velocity distributions are clearly different. In particular, the number of low-velocity sources is much higher in the M-star sample. We found no example of the sharply double-peaked CO line profile, which is evidence of a large, detached CO-shell, among the M-stars. About $10 \%$ of the C-stars show this phenomenon.
\end{abstract}

Key words. stars: AGB and post-AGB - mass loss - circumstellar matter - late-type - radio lines: stars

\section{Introduction}

It has been firmly established that mass loss from the surface is a very important process during the final stellar evolution of low- and intermediate-mass stars, i.e., on the asymptotic giant branch (AGB). The mass loss seems to occur irrespective of the chemistry $(\mathrm{C} / \mathrm{O}<1$ or $>1)$ or the variability pattern (irregular, semi-regular, or regular) of the star. Beyond these general conclusions the situation becomes more uncertain (Olofsson 1999). Of importance for comparison with mass loss models and for the understanding of AGB-stars in a broader context (e.g., their contribution to the chemical evolution of galaxies) is to establish the mass loss rate dependence on stellar parameters, such as main sequence mass, luminosity, effective

Send offprint requests to: H. Olofsson, e-mail: hans@astro.su.se temperature, pulsational pattern, metallicity, etc., and its evolution with time for individual sources. A crude picture has emerged where the average mass loss rate increases as the star evolves along the AGB, and where the final mass loss rate reached increases with the main sequence mass. In addition, there is evidence of time variable mass loss (Hale et al. 1997; Mauron \& Huggins 2000), and even highly episodic mass loss (Olofsson et al. 2000). There is a dependence on luminosity in the expected way, i.e., an increase with increasing luminosity, but it is uncertain how strong it is. The same applies to the effective temperature where a decrease with increasing temperature is expected. Regular pulsators clearly have higher mass loss rates than stars with less regular pulsation patterns. The dependence of the total mass loss rate on metallicity appears to be weak, but the dust mass loss rate decreases with decreasing 
metallicity. See Habing (1996) for a summary of evidence in favour of this general outline.

The mechanism behind the mass loss remains unknown, even though there are strong arguments in favour of a wind which is basically pulsation-driven, and where the highest mass loss rates and gas expansion velocities are reached through the addition of radiation pressure on dust (Höfner \& Dorfi 1997; Winters et al. 2000). A way to study this problem is to use samples of low mass loss rate stars for which stellar parameters can be reasonably estimated using traditional methods. These samples also contain objects with quite varying pulsational characteristics, and has, as it turned out, quite varying circumstellar characteristics. Olofsson et al. (1993) presented such a study of low mass loss rate $\mathrm{C}$-stars using $\mathrm{CO}$ multi-transition radio data. These data were subsequently analysed in more detail by Schöier \& Olofsson (2001) using a radiative transfer model. In the same spirit Kerschbaum \& Olofsson (1999) presented a major survey of $\mathrm{CO}$ radio line emission from irregularly variable (IRV) and semiregularly variable (SRV) M-type AGB-stars. They increased the number of IRVs (22 detections), in particular, and SRVs (43 detections) detected in circumstellar CO emission substantially ( $\approx 60 \%$ of the SRVs and all but one of the IRVs were detected for the first time). Young (1995) and Groenewegen et al. (1999) have made extensive surveys of short-period M-Miras.

In this paper we use the radiative transfer method of Schöier $\&$ Olofsson (2001) to estimate reasonably accurate mass loss rates and gas expansion velocities for the Kerschbaum \& Olofsson (1999) sample. Comparisons between these properties and other stellar properties are done, as well as comparisons with the results for the $\mathrm{C}$-star sample.

\section{The sample}

The original sample consisted of all IRV and SRV AGB-stars of spectral type M (determined by spectral classification, or using the IRAS LRS spectra) in the General Catalogue of Variable Stars [GCVS; Kholopov (1990)] with an IRAS quality flag 3 in the 12,25 , and $60 \mu \mathrm{m}$ bands. From this sample we selected for the CO radio line observations objects with IRAS $60 \mu \mathrm{m}$ fluxes, $S_{60}$, typically $\gtrsim 3$ Jy. Since $S_{60}$ for a star with a luminosity of $4000 L_{\odot}$ (see below) and a temperature of $2500 \mathrm{~K}$ is $34 \mathrm{Jy}$, $6 \mathrm{Jy}$, and $1.4 \mathrm{Jy}$ at a distance of $100 \mathrm{pc}, 250 \mathrm{pc}$, and $500 \mathrm{pc}$, respectively, we added a colour selection criterium. Only stars redder than $1.2 \mathrm{mag}$ in the IRAS [12]-[25] colour were observed, thus biasing the sample towards stars with detectable circumstellar dust envelopes. There is a possibility that stars with detectable gas mass loss rates, but with very little circumstellar dust, were missed due to this. About $50 \%$ of the objects, i.e., 109 sources, were subsequently searched for circumstellar radio line emission.

The $\mathrm{CO}(J=1 \rightarrow 0,2 \rightarrow 1,3 \rightarrow 2$, and $4 \rightarrow 3)$ data which are used as the observational constraints for the mass loss rate determinations in this paper were presented in Kerschbaum \& Olofsson (1999). They were obtained using the $20 \mathrm{~m}$ telescope at Onsala Space Observatory (OSO), Sweden, the $15 \mathrm{~m}$ Swedish-ESO Submillimetre Telescope (SEST) on La Silla, Chile, the IRAM $30 \mathrm{~m}$ telescope on Pico Veleta, Spain, and the James Clerk Maxwell Telescope on Mauna Kea, Hawaii. A few additional sources were observed at OSO in May 2000, see Sect. 3. In total, 69 stars were detected, 22 IRVs and 47 SRVs, and 45 were detected in at least two transitions, and 20 in at least three transitions. The detection rate was rather high, about $60 \%$, and the conclusions drawn in this paper should be representative for the stars in our sample.

The distances, presented in Table 3, were estimated using an assumed bolometric luminosity of $4000 L_{\odot}$. This value was chosen in agreement with the typical values derived by Kerschbaum et al. (1997) and Barthès et al. (1999) for objects with similar properties. Morover, the 13 objects in our sample having Hipparcos parallax errors better than $20 \%$ have a mean luminosity of $4200 L_{\odot}$ (with a standard deviation of $1900 L_{\odot}$, i.e., consistent with the assumed luminosity and the parallax uncertainty). For a statistical study of a large sample of stars these distance estimates are adequate (and they were used also for stars with reliable Hipparcos distances to avoid systematic differences), although the distance estimate for an individual star has a rather large uncertainty.

The apparent bolometric fluxes were obtained by integrating the spectral energy distributions ranging from the visual data over the near-infrared to the IRAS-range (Kerschbaum \& Hron 1996; Kerschbaum 1999).

\section{Observations}

A few additional sources were observed at OSO in May 2000 with the same instrumental setup as used by Kerschbaum \& Olofsson (1999). Relevant information on the instrumental setup, and the method used to derive the line profile properties and the upper limits can be found in Kerschbaum \& Olofsson (1999). A summary of the observational results are given in Table 1 (where we give the velocity-integrated intensities, $I_{\mathrm{mb}}$, and antenna temperatures, $T_{\mathrm{mb}}$, in main beam brightness scale, the stellar velocities as heliocentric, $v_{\text {hel }}$, and Local Standard of Rest, $v_{\mathrm{LSR}}$, velocities, and the gas expansion velocity, $v_{\mathrm{e}}$ ), and the detections are presented in Fig. 1.

\section{Radiative transfer}

We have chosen to use the Monte Carlo method to determine the excitation of the $\mathrm{CO}$ molecules as a function of distance from the central star (Bernes 1979). This is a versatile method, close to the physics, which has been applied to $\mathrm{CO}$ radio and far-IR line emission (Crosas \& Menten 1997; Ryde et al. 1999; Schöier et al. 2002), CO near-IR line scattering (Ryde \& Schöier 2001), and to the $\mathrm{OH} 1612 \mathrm{MHz}$ maser emission (van Langevelde \& Spaans 1993). Schöier \& Olofsson (2000, 2001) applied it to CO radio line data of sample of AGB carbon stars, and the results suggest that the modelling of circumstellar $\mathrm{CO}$ emission is one of the most reliable methods for estimating mass loss rates. The code has been benchmark tested together with a number of other radiative transfer codes (van Zadelhoff et al. 2002). Below we give a brief introduction to the modelling work, while for details we refer to Schöier \& Olofsson (2001). 
Table 1. $\mathrm{CO}(J=1 \rightarrow 0)$ results at OSO in May 2000.

\begin{tabular}{|c|c|c|c|c|c|c|c|c|c|}
\hline GCVS4 & IRAS & Var. & $\begin{array}{c}I_{\mathrm{mb}} \\
{\left[\mathrm{K} \mathrm{km} \mathrm{s}^{-1}\right]}\end{array}$ & $\begin{array}{l}T_{\mathrm{mb}} \\
{[\mathrm{K}]}\end{array}$ & $\begin{array}{c}v_{\text {hel }} \\
{\left[\mathrm{km} \mathrm{s}^{-1}\right]}\end{array}$ & $\begin{array}{c}v_{\mathrm{LSR}} \\
{\left[\mathrm{km} \mathrm{s}^{-1}\right]}\end{array}$ & $\begin{array}{c}v_{\mathrm{e}} \\
{\left[\mathrm{km} \mathrm{s}^{-1}\right]}\end{array}$ & $\mathrm{Q}^{1}$ & $\mathrm{C}$ \\
\hline CX Cas & $02473+6313$ & $\mathrm{SRa}$ & & & & & & 5 & IS-lines \\
\hline DP Ori & $05588+1054$ & $\mathrm{SRb}$ & $<1.3$ & & & & & 5 & \\
\hline Z Cnc & $08196+1509$ & $\mathrm{SRb}$ & $<0.7$ & & & & & 5 & \\
\hline RT Cnc & $08555+1102$ & $\mathrm{SRb}$ & $<0.5$ & & & & & 5 & \\
\hline SX Leo & $11010-0256$ & $\mathrm{SRb}$ & $<0.9$ & & & & & 5 & \\
\hline AF Leo & $11252+1525$ & $\mathrm{SRb}$ & $<0.7$ & & & & & 5 & \\
\hline AY Vir & $13492-0325$ & $\mathrm{SRb}$ & $<0.8$ & & & & & 5 & \\
\hline RY CrB & $16211+3057$ & $\mathrm{SRb}$ & 0.81 & 0.084 & 20.4 & 39.0 & 6.1 & 3 & \\
\hline CX Her & $17086+2739$ & $\mathrm{SRb}$ & $<0.5$ & & & & & 5 & \\
\hline IQ Her & $18157+1757$ & $\mathrm{SRb}$ & $<0.5$ & & & & & 5 & \\
\hline V988 Oph & $18243-0352$ & $\mathrm{SRb}$ & $<0.6$ & & & & & 5 & \\
\hline V585 Oph & $18247+0729$ & $\mathrm{SRb}$ & $<1.4$ & & & & & 5 & \\
\hline SY Lyr & $18394+2845$ & $\mathrm{SRb}$ & 1.4 & 0.18 & 39.1 & 58.9 & 4.5 & 2 & \\
\hline MZ Her & $18460+1903$ & $\mathrm{SRb}$ & $<0.6$ & & & & & 5 & \\
\hline V858 Aql & $19267+0345$ & $\mathrm{Lb}$ & $<2.8$ & & & & & 5 & \\
\hline AF Cyg & $19287+4602$ & $\mathrm{SRb}$ & 0.42 & 0.082 & -15.2 & 1.6 & 4.2 & 3 & \\
\hline V1172 Cyg & $19562+3304$ & $\mathrm{Lb}$ & & & & & & 5 & IS-lines \\
\hline V590 Cyg & $21155+4529$ & $\mathrm{Lb}$ & & & & & & 5 & IS-lines \\
\hline V655 Cyg & $21420+4746$ & $\mathrm{SRa}$ & & & & & & 5 & IS-lines \\
\hline RX Lac & $22476+4047$ & $\mathrm{SRb}$ & 1.3 & 0.28 & -26.5 & -15.8 & 3.5 & 2 & \\
\hline
\end{tabular}

${ }^{1}$ Quality parameter: 5 (non-det.), 4 (tent. det.), 3 (det., low S/N), 2 (det., good S/N), 1 (det., high S/N).
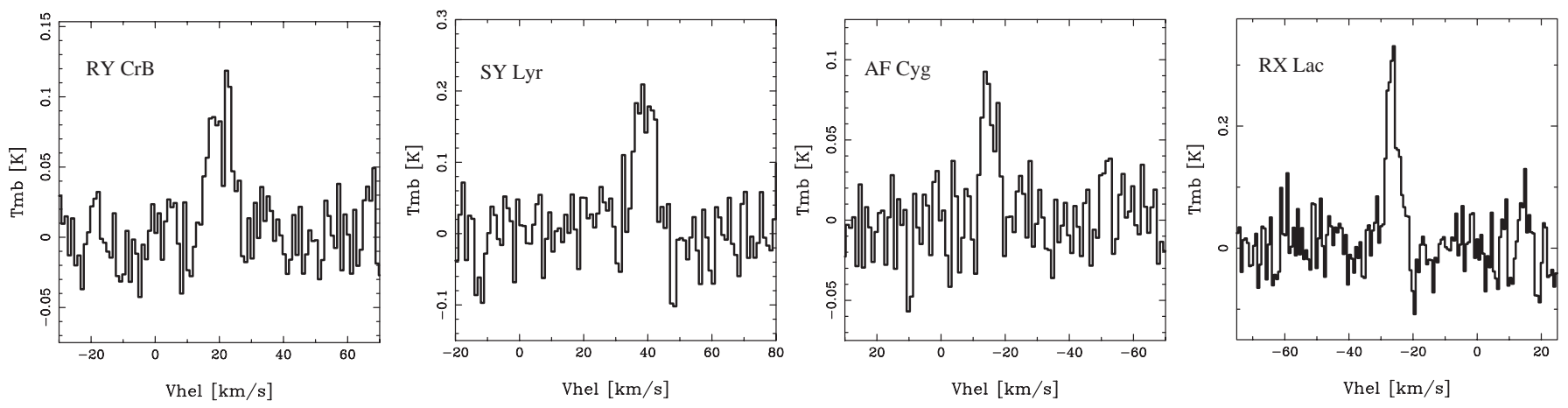

Fig. 1. $\mathrm{CO}(J=1 \rightarrow 0)$ detections at OSO in May 2000.

\subsection{The CO molecule}

In the excitation analysis of the $\mathrm{CO}$ molecule we used 40 rotational levels in each of the ground and first excited states. Excitation to the $v=2$ state can be ignored, since the $v=1$ state is not well populated. The radiative transition probabilities and energy levels were taken from Chandra et al. (1996). The collisional rate coefficients $\left(\mathrm{CO}-\right.$ para- $\left.\mathrm{H}_{2}\right)$ for rotational transitions are based on the results in Flower \& Launay (1985). These are further extrapolated for $J>11$ and for temperatures higher than $250 \mathrm{~K}$. We neglect collisional transitions between the vibrational states because of the low densities and the relatively low temperatures.

Recently, Flower (2001) presented revised and extended collisional rates for $\mathrm{CO}-\mathrm{H}_{2}$. Individual rates are generally different from those previously published in Flower \& Launay (1985), with discrepancies as large as a factor of two in some cases. In addition, for temperatures above $400 \mathrm{~K}$ the rates from Schinke et al. (1985) were used and further extrapolated to include transitions up to $J=40$. To test the effects of the adopted set of collisional rates a number of test cases with the new rates assuming an $\mathrm{H}_{2}$ ortho-to-para ratio of three were run. We found that for the relatively low mass loss rate stars of interest here, where excitation of $\mathrm{CO}$ from radiation dominates over that from collisions with $\mathrm{H}_{2}$, the adopted set of collisional rates is only of minor importance.

\subsection{The circumstellar model}

\subsubsection{The geometry and kinematics}

We have adopted a relatively simple, yet realistic, model for the geometry and kinematics of the CSE. It is assumed that the mass loss is isotropic and constant with time. As will be shown below the median mass loss rate is $2 \times 10^{-7} M_{\odot} \mathrm{yr}^{-1}$ and the median gas expansion velocity is $7.0 \mathrm{~km} \mathrm{~s}^{-1}$ for our sample. This results in an extent of the $\mathrm{CO}$ envelope of 
about $2 \times 10^{16} \mathrm{~cm}$ (see Sect. 4.2.3), and the corresponding time scale is about $1000 \mathrm{yr}$. There is now some evidence for mass loss rate modulations of AGB-stars on this time scale (Mauron \& Huggins 2000; Marengo et al. 2001), and therefore the assumption of a constant mass loss rate may be questionable, and this must be kept in mind when interpreting the results. The gas expansion velocity, $v_{\mathrm{e}}$, is assumed to be constant with radius. This ignores the more complex situation in the inner part of the CSE, but the emission in the CO lines detected by radio telescopes mainly comes from the external part. We further assume that the hydrogen is in molecular form in the region probed by the CO emission (Glassgold \& Huggins 1983). As a consequence of these assumptions the $\mathrm{H}_{2}$ number density follows an $r^{-2}$-law.

In the case of low mass loss rate objects the inner radius of the CSE will have an effect on the model intensities. The reason is that radiative excitation plays a role in this case and the absorption of pump photons at $4.6 \mu \mathrm{m}$ depends (sensitively) on this choice. We set the inner radius to $1 \times 10^{14} \mathrm{~cm}\left(\sim 3 R_{*}\right)$, i.e., generally beyond both the sonic point and the dust condensation radius. The uncertainty in the mass loss rate estimate introduced by this assumption is discussed in Sect. 4.3. Strictly, speaking the assumption of a constant expansion velocity from this inner radius is very likely not correct. An acceleration region will enhance the radiative excitation and hence may have an effect on the estimated mass loss rate. However, as will be shown in Sect. 4.3, the dependence on the inner radius is rather modest, suggesting that the properties of the inner CSE are not crucial for the mass loss rate determination. We have therefore refrained from introducing yet another parameter, i.e., a velocity law parameter.

In addition to thermal broadening of the lines microturbulent motions contribute to the Doppler broadening of the local line width. We assume a turbulent velocity width, $v_{\mathrm{t}}$, of $0.5 \mathrm{~km} \mathrm{~s}^{-1}$ throughout the entire CSE, i.e., the same value as used by Schöier \& Olofsson (2001) (for reference the thermal width of CO is about $\left.0.3\left(T_{\mathrm{k}} / 100\right)^{0.5} \mathrm{~km} \mathrm{~s}^{-1}\right)$. This can be an important parameter for low mass loss rate objects since it affects the radial optical depths and hence the effectiveness of the radiative excitation. The constraints on this parameter are rather poor. The most thorough analysis in this connection is the one by Huggins \& Healy (1986). They modelled in detail the circumstellar CO line self-absorption in the high mass loss rate carbon star IRC+10216 and derived a value of $0.9 \mathrm{~km} \mathrm{~s}^{-1}$. In Sect. 4.3 we discuss the uncertainty in the mass loss rate estimate introduced by this parameter.

\subsubsection{Heating and cooling processes}

We determine the kinetic gas temperature in the CSE by taking into account a number of heating and cooling processes (Groenewegen 1994). The primary heating process is the viscous heating due to the dust streaming through the gas medium. A drift velocity between the gas and the dust is calculated assuming a dust-driven wind (Kwok 1975), but for the low mass loss rate stars in this study the radiation pressure on dust may not be very efficient, i.e., the driving of the gas may be due to something else. However, as will be explained below, the gas-dust heating term is nevertheless very uncertain, and we use it as a free parameter in our model. Additional heating is due to the photoelectric effect, i.e., heating by electrons ejected from the grains by cosmic rays (Huggins et al. 1988), but for our low mass loss rate stars this has a negligible effect inside the $\mathrm{CO}$ envelope.

There are three major cooling processes, adiabatic expansion of the gas, $\mathrm{CO}$ line cooling, and $\mathrm{H}_{2} \mathrm{O}$ line cooling. The $\mathrm{CO}$ line cooling is taken care of self-consistently by calculating its magnitude after each iteration using the expression of Crosas \& Menten (1997). $\mathrm{H}_{2} \mathrm{O}$ line cooling is estimated using the results from Neufeld \& Kaufman (1993). They calculated the $\mathrm{H}_{2} \mathrm{O}$ excitation using an escape probability method and estimated the radiative cooling rates for a wide range of densities and temperatures. The $\mathrm{H}_{2} \mathrm{O}$ abundance is set to $2 \times 10^{-4}$ and the envelope sizes used are based on the results of Netzer \& Knapp (1987). In addition, $\mathrm{H}_{2}$ line cooling is taken into account (Groenewegen 1994), but this has negligible effect in the regions of interest here.

When solving the energy balance equation a number of (uncertain) parameters describing the dust are introduced. Following Schöier \& Olofsson (2001) we assume that the $Q_{\mathrm{p}, \mathrm{F}}$-parameter, i.e., the flux-averaged momentum transfer effeciency from the dust to the gas, is equal to 0.03 (see Habing et al. 1994 for details), and define a new parameter which contains the other dust parameters,

$h=\left[\frac{\psi}{0.01}\right]\left[\frac{2.0 \mathrm{~g} \mathrm{~cm}^{-3}}{\rho_{\mathrm{gr}}}\right]\left[\frac{0.05 \mu \mathrm{m}}{a_{\mathrm{gr}}}\right]$,

where $\psi$ is the dust-to-gas mass ratio, $\rho_{\mathrm{gr}}$ the dust grain density, and $a_{\mathrm{gr}}$ its radius. The normalized values are the ones used to fit the CO radio line emission of IRC+10216 using this model (Schöier \& Olofsson 2001), i.e., $h=1$ for this object.

\subsubsection{The $\mathrm{CO}$ fractional abundance distribution}

We assume that the initial fractional abundance of $\mathrm{CO}$ with respect to $\mathrm{H}_{2}, f_{\mathrm{CO}}$, is $2 \times 10^{-4}$, which is the same value as used by Kahane \& Jura (1994) in their analysis of CO radio line emission from M-stars. This is essentially a free parameter, although its upper limit is given by the abundance of $\mathrm{C}$ (i.e., $7 \times 10^{-4}$ is the upper limit for a solar $\mathrm{C}$ abundance). Due to photodissociation by the interstellar radiation field the $\mathrm{CO}$ abundance starts to decline rapidly at a radius, which, for not too low mass loss rates, depends on the mass loss rate. Calculations, taking into account dust-, self- and $\mathrm{H}_{2}$-shielding, and chemical fractionation, have been performed by Mamon et al. (1988) and Doty \& Leung (1998). Here we use the results of Mamon et al. (1988) in the way adopted by Schöier \& Olofsson (2001).

An approximate expression for the photodissociation radius, $r_{\mathrm{p}}$, consists of two terms, the unshielded size due to the expansion, which is independent of the mass loss rate, and the size due to the self-shielding, which scales roughly as $\left(f_{\mathrm{CO}} \dot{M}\right)^{0.5}$ (Schöier \& Olofsson 2001). These terms are equal at a mass loss rate of about $4 \times 10^{-8}\left(v_{\mathrm{e}} / 7\right)^{2} M_{\odot} \mathrm{yr}^{-1}$ for the adopted CO abundance $\left(v_{\mathrm{e}}\right.$ is given in $\left.\mathrm{km} \mathrm{s}^{-1}\right)$. That is, self-shielding plays a role for essentially all of our objects. We note that for low 
Table 2. The effect on the velocity-integrated model intensities (in percent), due to changes in various parameters. Three model stars with nominal mass loss rate and gas expansion velocity characteristics typical for our sample are used. They lie at a distance of $250 \mathrm{pc}$, and have luminosities of $4000 L_{\odot}$ and blackbody temperatures of $2500 \mathrm{~K}$. The nominal CSE parameters are $h=0.2, r_{\mathrm{i}}=2 \times 10^{14} \mathrm{~cm} v_{\mathrm{t}}=0.5 \mathrm{~km} \mathrm{~s}{ }^{-1}$, and $f_{\mathrm{CO}}=2 \times 10^{-4}$. The $\mathrm{CO}(J=1 \rightarrow 0), \operatorname{CO}(J=2 \rightarrow 1)$, and $\mathrm{CO}(J=3 \rightarrow 2)$ lines are observed with beam widths of $33^{\prime \prime}, 23^{\prime \prime}$, and $14^{\prime \prime}$, respectively. The model integrated line intensities, $I$, are given for the nominal parameters.

\begin{tabular}{|c|c|c|c|c|c|c|c|c|c|c|}
\hline \multirow[b]{2}{*}{ Parameter } & \multirow[b]{2}{*}{ Change } & \multicolumn{3}{|c|}{$4 \times 10^{-8} M_{\odot} \mathrm{yr}^{-1}, 5 \mathrm{~km} \mathrm{~s}^{-1}$} & \multicolumn{3}{|c|}{$1 \times 10^{-7} M_{\odot} \mathrm{yr}^{-1}, 7 \mathrm{~km} \mathrm{~s}^{-1}$} & \multicolumn{3}{|c|}{$5 \times 10^{-7} M_{\odot} \mathrm{yr}^{-1}, 10 \mathrm{~km} \mathrm{~s}^{-1}$} \\
\hline & & $1-0$ & $2-1$ & $3-2$ & $\overline{1-0}$ & $2-1$ & $3-2$ & $1-0$ & $2-1$ & $3-2$ \\
\hline$I\left[\mathrm{~K} \mathrm{~km} \mathrm{~s}^{-1}\right]$ & & 0.043 & 0.39 & 1.80 & 0.25 & 1.53 & 4.40 & 2.64 & 6.61 & 13.00 \\
\hline \multirow[t]{2}{*}{$\dot{M}$} & $-50 \%$ & -75 & -75 & -70 & -80 & -75 & -60 & -55 & -50 & -45 \\
\hline & $+100 \%$ & +530 & +300 & +150 & +360 & +140 & +75 & +140 & +90 & +75 \\
\hline \multirow[t]{2}{*}{$L$} & $-50 \%$ & +80 & +40 & 0 & +70 & -5 & -30 & -15 & -35 & -40 \\
\hline & $+100 \%$ & -35 & -30 & -20 & -50 & -20 & +10 & +10 & +30 & +35 \\
\hline \multirow[t]{2}{*}{$h$} & $-50 \%$ & +5 & +10 & +5 & +30 & +5 & -5 & -5 & -20 & -30 \\
\hline & $+100 \%$ & -5 & -10 & -5 & -15 & -10 & 0 & -5 & +15 & +30 \\
\hline \multirow[t]{2}{*}{$r_{\mathrm{p}}$} & $-50 \%$ & -75 & -75 & -65 & -80 & -70 & -50 & -60 & -35 & -20 \\
\hline & $+100 \%$ & +300 & +170 & +80 & +250 & +80 & +35 & +60 & +15 & +5 \\
\hline \multirow[t]{2}{*}{$r_{\mathrm{i}}$} & $-50 \%$ & +15 & +20 & +10 & +25 & 0 & -10 & -5 & -5 & 0 \\
\hline & $+100 \%$ & -10 & -10 & -10 & -15 & -5 & 0 & +5 & +5 & 0 \\
\hline \multirow[t]{2}{*}{$v_{\mathrm{t}}$} & $-50 \%$ & +15 & +25 & +5 & +40 & 0 & -15 & -5 & -5 & -5 \\
\hline & $+100 \%$ & -20 & -20 & -10 & -30 & -15 & +5 & +15 & +15 & +15 \\
\hline
\end{tabular}

mass loss rate objects the spatial extent of the $\mathrm{CO}$ envelope is particularly important since the spatial extent of the $\mathrm{CO}$ line emission is limited by this, and not by excitation, Sect. 4.3.

\subsubsection{The radiation fields}

The radiation field is provided by two sources. The central radiation emanates from the star, and was estimated from a fit to the spectral energy distribution (SED), usually by assuming two blackbodies, one representing the direct stellar radiation and one the dust-processed radiation (Kerschbaum \& Hron 1996). The dust mass loss rates of our sample stars are low enough that the latter can be ignored. The temperatures derived are given in Table 3 . The stellar blackbody temperature $T_{\mathrm{bb}}$ derived in this manner is generally about $500 \mathrm{~K}$ lower than the effective temperature of the star (Kerschbaum \& Hron 1996). The second radiation field is provided by the cosmic microwave background radiation at $2.7 \mathrm{~K}$.

\subsection{Dependence on parameters}

We have checked the sensitivity of the calculated intensities on the assumed parameters for a set of model stars. The model stars have nominal mass loss rate and gas expansion velocity combinations which are characteristic of our sample: $\left(4 \times 10^{-8} M_{\odot} \mathrm{yr}^{-1}, 5 \mathrm{~km} \mathrm{~s}^{-1}\right),\left(1 \times 10^{-7} M_{\odot} \mathrm{yr}^{-1}, 7 \mathrm{~km} \mathrm{~s}^{-1}\right)$, and $\left(5 \times 10^{-7} M_{\odot} \mathrm{yr}^{-1}, 10 \mathrm{~km} \mathrm{~s}^{-1}\right)$. They are placed at a distance of $250 \mathrm{pc}$ (a typical distance of our stars), and the nominal values of the other parameters are $L=4000 L_{\odot}, T_{\mathrm{bb}}=2500 \mathrm{~K}, h=0.2$ (the value adopted for the majority of our stars, Sect. 5.2), $r_{\mathrm{i}}=2 \times 10^{14} \mathrm{~cm}$ (this is twice the inner radius used in the modelling), $v_{\mathrm{t}}=0.5 \mathrm{~km} \mathrm{~s}^{-1}, f_{\mathrm{CO}}=2 \times 10^{-4}$, and $r_{\mathrm{p}}$ is calculated from the photodissociation model (Sect. 4.2.3). The $\mathrm{CO}(J=1 \rightarrow 0), \mathrm{CO}(J=2 \rightarrow 1)$, and $\mathrm{CO}(J=3 \rightarrow 2)$ lines are observed with beam widths of $33^{\prime \prime}, 23^{\prime \prime}$, and $14^{\prime \prime}$, respectively. These are characteristic angular resolutions of our observations. Note that, to some extent, the presented results are dependent on the assumed angular resolution since resolution effects may play a role. We change all parameters (except the expansion velocity) by $-50 \%$ and $+100 \%$ and calculate the velocity-integrated intensities.

The results are summarized in Table 2 in terms of percentage changes. Although the dependences are somewhat complicated there are some general trends. The line intensities are sensitive to changes in the mass loss rate, more the lower the mass loss rate, and hence are sensitive measures of this property. There is a dependence on luminosity, in particular for low$J$ lines for low mass loss rates and for high- $J$ lines for higher mass loss rates. The dependence on the (uncertain) $h$-parameter is fortunately rather weak. The dependence on the photodissociation radius is substantial, in particular for the low- $J$ lines and for low mass loss rates. The dependence on the inner radius is weak, and so is the dependence on the turbulent velocity width. Thus, we conclude that for our objects the $\mathrm{CO}$ radio line intensities are good measures of the mass loss rate, but it shall be kept in mind that they are rather dependent on the uncertain photodissociation radius and, to some extent, on the assumed luminosity. A similar sensitivity analysis for C-stars were done by Schöier \& Olofsson (2001), and studies of the parameter dependence were done by Kastner (1992) and Kwan \& Webster (1993).

There is also a dependence on the adopted CO abundance. For the low mass loss rates considered here $\left(\lesssim 5 \times 10^{-7} M_{\odot} \mathrm{yr}^{-1}\right)$ a constant product $f_{\mathrm{CO}} \dot{M}$ produces the same model line intensities. Hence, mass loss rates for a different value of $f_{\mathrm{CO}}$ are easily obtained. The reason for this behaviour is that the size of the emitting region is photodissociation limited rather than excitation limited, i.e., for our objects it scales roughly as $\left(f_{\mathrm{CO}} \dot{M}\right)^{0.5}$, see Sect. 4.2.3. Furthermore, the energy levels are to a large extent radiatively excited, i.e., the density plays less of a role for the excitation. Therefore, since the emission is optically thin, 

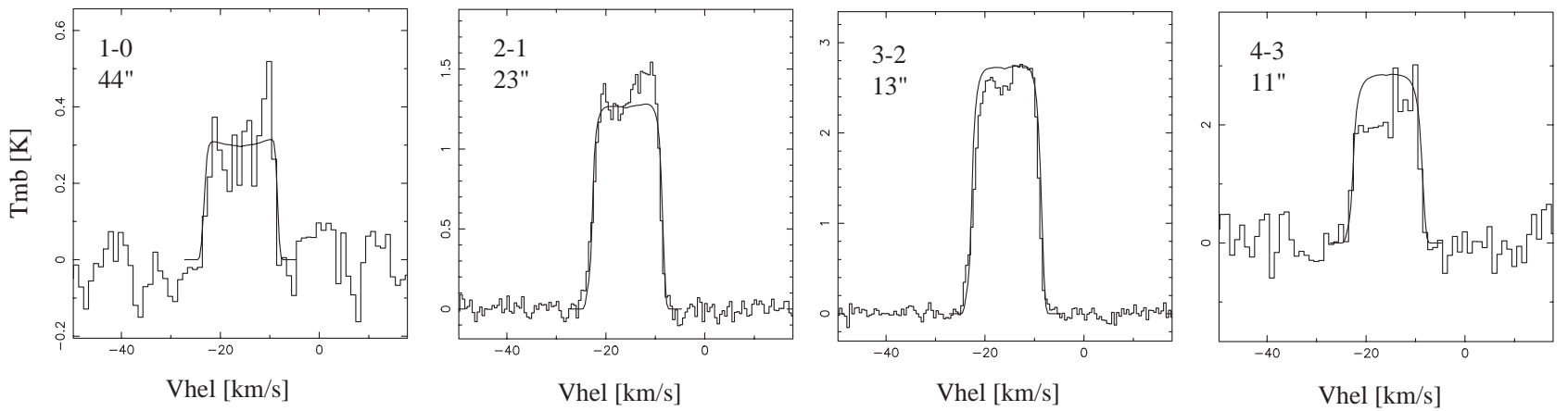

Fig. 2. SW Vir $\operatorname{CO}(J=1 \rightarrow 0$ and $2 \rightarrow 1)$ spectra obtained with the SEST, and $\operatorname{CO}(J=3 \rightarrow 2$ and $4 \rightarrow 3)$ spectra obtained with the JCMT (histograms). The line profiles from the best-fit model are shown as solid, thin lines (the beam size is given in each panel).

a change in the abundance must be compensated by an equal, but opposite, change in the mass loss rate to keep the calculated intensities unchanged.

An additional uncertainty is due to the somewhat crude treatment of $\mathrm{H}_{2} \mathrm{O}$ line cooling. The modelling shows that this cooling process has an effect on the temperature structure of the CSE. However, it is found to be of importance only in the inner warm and dense part of the CSE where $\mathrm{H}_{2} \mathrm{O}$ is abundant. The size of the $\mathrm{H}_{2} \mathrm{O}$ envelope is only about a tenth of the $\mathrm{CO}$ envelope. This small region contributes only marginally to the line intensities of the observed low- $J$ transitions (see for instance the weak dependence on $r_{\mathrm{i}}$ ).

\section{Model results}

\subsection{Best-fit model strategy}

The radiative transfer analysis produces model brightness distributions. These are convolved with the appropriate beams to allow a direct comparison with the observed velocity-integrated line intensities and to search for the best fit model. There are two remaining parameters to vary in this fitting procedure, the mass loss rate $\dot{M}$ and the $h$-parameter. These two parameters were varied until the best-fit model was found. The quality of a fit was quantified using the chi-square statistic,

$\chi_{\mathrm{red}}^{2}=\frac{1}{N-p} \sum_{i=1}^{\mathrm{N}} \frac{\left.\left[I_{\mathrm{mod}, i}-I_{\mathrm{obs}, i}\right)\right]^{2}}{\sigma_{i}^{2}}$,

where $I$ is the total integrated line intensity, $\sigma_{i}$ the uncertainty in observation $i, p$ the number of free parameters, and the summation is done over all independent observations $N$. The errors in the observed intensities are always larger than the calibration uncertainty of $\sim 20 \%$. We have chosen to adopt $\sigma_{i}=0.2 I_{\mathrm{obs}, i}$ to put equal weight on all lines, irrespective of the $\mathrm{S} / \mathrm{N}$-ratio. Initially a grid, centered on $\dot{M}=10^{-7} M_{\odot} \mathrm{yr}^{-1}$ and $h=0.1$, with step sizes of $50 \%$ in $\dot{M}$ and $100 \%$ in $h$ was used to locate the $\chi^{2}$-minimum. The final parameters were obtained by decreasing the step size to $25 \%$ in $\dot{M}$ and $50 \%$ in $h$ and by interpolating between the grid points. The final chi-square values for stars observed in more than one transition are given in Table 3. The line profiles were not used to discriminate between models, but differences between model and observed line profiles are discussed in Sect. 6.6.
In general, the model results fit rather well the observed data as can be seen from the chi-square values. For instance, we reproduce the very high $(J=2 \rightarrow 1) /(J=1 \rightarrow 0)$ intensity ratios reported for these objects, 4.2 on average and ratios of 10 are not uncommon (Kerschbaum \& Olofsson 1999). Table 2 gives the integrated line intensities of our model stars in Sect. 4.3. These give an indication of how the line intensities depend on the mass loss rate. In particular, one should note the large intensity ratios for low mass loss rates: $I(2-1) / I(1-0)$ equals about 9,5 , and 3 , and $I(3-2) / I(1-0)$ equals about 42,13 , and 5 for $4 \times 10^{-8} M_{\odot} \mathrm{yr}^{-1}, 1 \times 10^{-7} M_{\odot} \mathrm{yr}^{-1}$, and $5 \times 10^{-7} M_{\odot} \mathrm{yr}^{-1}$, respectively. In Fig. 2 we present the observational data of SW Vir and the best-fit model results.

\subsection{The h-parameter}

The intensity ratios between lines of different excitation requirements are sensitive to the temperature structure. Therefore, we initially used stars with three, or more, transitions observed to estimate $h$. In total, 16 objects fulfil this criterium and the resulting model fits are rather good as shown by the $\chi_{\text {red }}^{2}$-values, Table 3 . The derived $h$-values have a mean of 0.24 and a median of 0.21 . The scatter in the derived $h$-values is rather large, and there is no apparent trend with the density measure $\dot{M} / v_{\mathrm{e}}$, Fig. 3. We also determined, in the same way, the $h$-values for those stars with only two observed transitions ( 25 objects), and the result was a mean of 0.22 and a median of 0.1 . There is no trend with the density measure for these objects either, and the scatter is large, Fig. 3. As outlined above the line intensities of low mass loss rate objects are not particularly sensitive to $h$, and this very likely contributes to the large scatter in the derived values. The median value is clearly lower for the sources observed in only two transitions. This is probably due to a systematic effect. Pointing and calibration problems tend to affect more the higher-frequency lines, and will, on average, lead to too low observed intensities. A low intensity ratio between a higher-frequency and a lower-frequency line can be accomodated in the model only by "cooling" the envelope, i.e., by lowering $h$. A decrease in $h$ must be compensated by an increase in $\dot{M}$ to preserve the line intensities. To avoid this systematic effect we used $h=0.2$ for all objects observed in less than three transitions. We conclude that the derived mass loss rates are not sensitively dependent on this choice, unless 


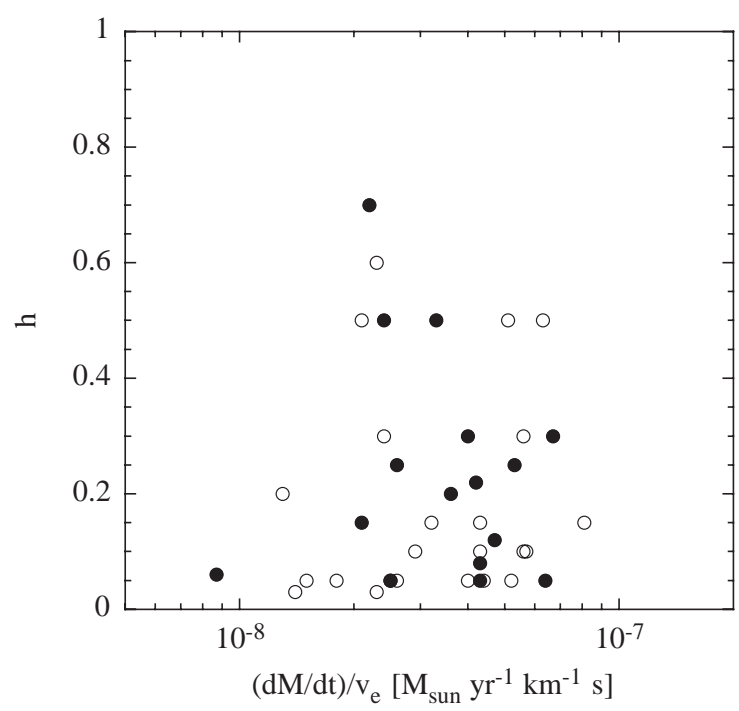

Fig. 3. The $h$-parameters derived from the radiative transfer analysis plotted against the density measure $\dot{M} / v_{\mathrm{e}}$. Objects with three or more transitions observed are marked with filled circles, while those with only two transitions observed are marked with open circles.

the true $h$ is considerably lower than this value. We note from the results of Schöier \& Olofsson (2001) that an increase in $h$ will lead to a decrease in $\dot{M}$, and vice versa.

Schöier \& Olofsson (2001) found an $h$-value of about 1 for the high mass loss rate carbon stars, and a trend of decreasing values towards lower mass loss rates, reaching an average of about 0.2 in the mass loss rate range of our stars. The presumed difference in grain density between carbon grains $\left(2.0 \mathrm{~g} \mathrm{~cm}^{-3}\right)$ and silicate grains $\left(3.0 \mathrm{~g} \mathrm{~cm}^{-3}\right)$ means that for the same grain size our $h$-value of 0.2 indicates a dust-to-gas mass ratio which is 1.5 times higher, i.e., $3 \times 10^{-3}$, in the CSEs of M-type stars. However, the uncertainties are such that we can only conclude that both the M-type and C-type CSEs due to low mass loss rates appear to have dust properties significantly different from those of C-type CSEs due to high mass loss rates (however, all mass loss rate determinations are made using the same value for the flux-averaged dust momentum transfer efficiency, which determines the gas-dust drift velcoity and hence affects the heating of the CSE, while in reality it may depend on the mass loss rate). We can also conclude that the gas-CSEs due to low mass loss rates are cooler than expected from a simple extrapolation of the results for IRC+10216 (Groenewegen et al. 1998; Crosas \& Menten 1997; Schöier \& Olofsson 2000).

\subsection{The mass loss rates}

The estimated mass loss rates are given in Table 3 (rounded off to the number nearest to $1.0,1.3,1.5,2.0,2.5,3,4,5,6$, or 8 , i.e., these values are separated by about $25 \%$ ), and their distributions are shown in Fig. 4 (four sources with clearly peculiar line profiles are discussed separately in Sect. 6.7). We estimate that, within the adopted circumstellar model, the derived mass loss rates are uncertain by about $\pm 20 \%$ for those sources with three or more observed transitions, since the line intensities are very sensitive to changes in this parameter (see Table 2). The uncertainty increases when less than three transitions are observed, generally $\pm 50 \%$, but it may be as bad as a factor of a few for objects with low $h$-values. To this should be added the uncertainty due to the distance, the luminosity, the photodissociation radius, the fractional $\mathrm{CO}$ abundance, the collisional cross sections, and the pointing/calibration. Nevertheless, as a whole, we believe that these are the most accurate mass loss rates determined for these types of objects, but on an absolute scale they are uncertain by at least a factor of a few for an individual object. A comparison with the mass loss rates estimated by Knapp et al. (1998) for the six stars in common shows differences by less than a factor of two, except in the case of RT Vir for which we derive a five times higher value. Note that the mass loss rates given are not corrected for the He-abundance, i.e., they are molecular hydrogen mass loss rates.

The detailed radiative transfer presented here results in considerably higher mass loss rates than those obtained with the simpler analysis in Kerschbaum et al. (1996) and Kerschbaum \& Olofsson (1998). For the 43 objects in common we derive mass loss rates which are on average ten times higher for the same distances and $\mathrm{CO}$ abundance (the median difference is six). This confirms the conclusion by Schöier \& Olofsson (2001) that the formulae of Knapp \& Morris (1985) and Kastner (1992) lead to substantially underestimated mass loss rates for low mass loss rate objects. This is no surprise since both formulae were calibrated against IRC+10216 (for which $h=1$ ), and in the case of Knapp \& Morris (1985) an older CO photodissociation model was used.

\subsection{The gas expansion velocities}

The gas expansion velocities given in Table 3 are obtained in the model fits. Hence, they should be somewhat more accurate than the pure line profile fit results given in Kerschbaum \& Olofsson (1999), since for instance the effect of turbulent broadening is taken into account. The former are in general somewhat lower than the latter. We estimate the uncertainty to be of the order $\pm 10 \%$. The uncertainty is dominated by the $\mathrm{S} / \mathrm{N}$-ratio since the spectral resolution is in most cases more than adequate. A significant fraction of the sources has gas expansion velocities lower than $5 \mathrm{~km} \mathrm{~s}^{-1}$, and for these the assumption of a turbulent velocity width of $0.5 \mathrm{~km} \mathrm{~s}^{-1}$ will have some effect on the expansion velocity estimate. The gas expansion velocity distribution for the whole sample, as well as those of the IRVs and SRVs separately, are shown in Fig. 5 (excluding the double-component sources, Sect. 6.7).

\section{Discussion}

In this section we present and discuss a number of results based on the derived mass loss rates and gas expansion velocities. Extensive comparisons are made with the results of the C-type IRVs and SRVs (39 objects) analysed by Schöier \& Olofsson (2001) using the same methods as in this study. 
Table 3. Source parameters and model results.

\begin{tabular}{|c|c|c|c|c|c|c|c|c|c|c|}
\hline Source & $\begin{array}{l}\text { Var. } \\
\text { type }\end{array}$ & $\begin{array}{c}P \\
\text { [days] }\end{array}$ & $\begin{array}{r}D^{1} \\
{[\mathrm{pc}]}\end{array}$ & $\begin{array}{l}T_{\mathrm{bb}} \\
{[\mathrm{K}]}\end{array}$ & $\begin{array}{c}\dot{M} \\
{\left[10^{-7} M_{\odot} \mathrm{yr}^{-1}\right]}\end{array}$ & $\begin{array}{c}v_{\mathrm{e}} \\
{\left[\mathrm{km} \mathrm{s}^{-1}\right]}\end{array}$ & $\begin{array}{c}r_{\mathrm{p}} \\
{\left[10^{16} \mathrm{~cm}\right]}\end{array}$ & $h$ & $\chi_{\mathrm{red}}^{2}$ & $N$ \\
\hline BC And & $\mathrm{Lb}$ & & 450 & 2510 & 2.0 & 4.0 & 2.0 & & & 1 \\
\hline CE And & $\mathrm{Lb}$ & & 740 & 2720 & 5 & 10.5 & 2.5 & & & 1 \\
\hline RS And & $\mathrm{SRa}$ & 136 & 290 & 2620 & 1.5 & 4.4 & 1.6 & & 0.7 & 2 \\
\hline UX And & $\mathrm{SRb}$ & 400 & 280 & 2240 & 4 & 12.8 & 2.1 & & 1.9 & 2 \\
\hline TZ Aql & $\mathrm{Lb}$ & & 470 & 2460 & 1.0 & 4.8 & 1.3 & & & 1 \\
\hline V584 Aql & $\mathrm{Lb}$ & & 390 & 2340 & 0.5 & 2.2 & 1.2 & & & 1 \\
\hline $\mathrm{AB}$ Aqr & $\mathrm{Lb}$ & & 460 & 2580 & 1.3 & 4.2 & 1.5 & & & 1 \\
\hline SV Aqr & $\mathrm{Lb}$ & & 470 & 2180 & 3 & 8.0 & 2.1 & & 9.1 & 2 \\
\hline$\theta$ Aps & $\mathrm{SRb}$ & 119 & 110 & 2620 & 0.4 & 4.5 & 0.8 & & 9.8 & 2 \\
\hline T Ari & $\mathrm{SRa}$ & 317 & 310 & 2310 & 0.4 & 2.4 & 0.9 & & & 1 \\
\hline RX Boo & $\mathrm{SRb}$ & 340 & 110 & 2220 & 5 & 9.3 & 2.6 & & 1.4 & 2 \\
\hline RV Cam & $\mathrm{SRb}$ & 101 & 350 & 2570 & 2.5 & 5.8 & 2.0 & & 0.4 & 2 \\
\hline BI Car & $\mathrm{Lb}$ & & 430 & 2420 & 0.3 & 2.2 & 0.9 & & & 1 \\
\hline V744 Cen & $\mathrm{SRb}$ & 90 & 200 & 2750 & 1.3 & 5.3 & 1.5 & 0.05 & 6.0 & 3 \\
\hline SS Cep & $\mathrm{SRb}$ & 90 & 340 & 2580 & 6 & 10.0 & 2.7 & & 3.3 & 2 \\
\hline UY Cet & $\mathrm{SRb}$ & 440 & 300 & 2400 & 2.5 & 6.0 & 2.0 & 0.2 & 0.4 & 3 \\
\hline $\mathrm{CW}$ Cnc & $\mathrm{Lb}$ & & 280 & 2400 & 5 & 8.5 & 2.5 & 0.25 & 3.2 & 3 \\
\hline RY CrB & $\mathrm{SRb}$ & & 550 & 2340 & 4 & 5.7 & 2.5 & & & 1 \\
\hline R Crt & $\mathrm{SRb}$ & 160 & 170 & 2130 & 8 & 10.6 & 3.0 & 0.3 & 0.7 & 4 \\
\hline AF Cyg & $\mathrm{SRb}$ & & 300 & 2840 & 0.8 & 3.5 & 1.2 & & & 1 \\
\hline W Cyg & $\mathrm{SRb}$ & 131 & 130 & 2670 & 1.0 & 8.3 & 1.3 & & 0.7 & 2 \\
\hline U Del & $\mathrm{SRb}$ & 110 & 210 & 2720 & 1.5 & 7.5 & 1.5 & & 10.5 & 2 \\
\hline R Dor & $\mathrm{SRb}$ & 338 & 45 & 2090 & 1.3 & 6.0 & 1.4 & 0.7 & 1.6 & 3 \\
\hline AH Dra & $\mathrm{SRb}$ & 158 & 340 & 2680 & 0.8 & 6.4 & 1.1 & & & 1 \\
\hline CS Dra & $\mathrm{Lb}$ & & 370 & 2580 & 6 & 11.6 & 2.7 & 0.05 & 3.2 & 3 \\
\hline S Dra & $\mathrm{SRb}$ & 136 & 270 & 2230 & 4 & 9.6 & 2.2 & 0.3 & 0.5 & 3 \\
\hline SZ Dra & $\mathrm{Lb}$ & & 510 & 2580 & 6 & 9.6 & 2.7 & & & 1 \\
\hline TY Dra & $\mathrm{Lb}$ & & 430 & 2300 & 6 & 9.0 & 2.8 & & 1.0 & 2 \\
\hline UU Dra & $\mathrm{SRb}$ & 120 & 320 & 2260 & 5 & 8.0 & 2.5 & & 5.0 & 2 \\
\hline g Her & $\mathrm{SRb}$ & 89 & 100 & 2700 & 1.0 & 8.4 & 1.3 & & & 1 \\
\hline AK Hya & $\mathrm{SRb}$ & 75 & 210 & 2430 & 1.0 & 4.8 & 1.3 & 0.15 & 1.5 & 4 \\
\hline EY Нуа & $\mathrm{SRa}$ & 183 & 300 & 2400 & 2.5 & 11.0 & 1.8 & & 1.1 & 2 \\
\hline FK Hya & $\mathrm{Lb}$ & & 310 & 2630 & 0.6 & 8.7 & 1.0 & & & 1 \\
\hline FZ Hya & $\mathrm{Lb}$ & & 330 & 2460 & 2.0 & 7.8 & 1.6 & & 0.0 & 2 \\
\hline W Hya & $\mathrm{SRa}$ & 361 & 65 & 2090 & 0.7 & 6.5 & 1.0 & & & 1 \\
\hline RX Lac & $\mathrm{SRb}$ & & 250 & 2450 & 0.8 & 2.2 & 1.6 & & & 1 \\
\hline RW Lep & $\mathrm{SRa}$ & 150 & 400 & 2150 & 0.5 & 4.4 & 0.9 & & & 1 \\
\hline RX Lep & $\mathrm{SRb}$ & 60 & 150 & 2660 & 0.5 & 3.5 & 1.0 & & 0.2 & 2 \\
\hline SY Lyr & $\mathrm{SRb}$ & & 640 & 2410 & 6 & 4.6 & 3.6 & & & 1 \\
\hline TU Lyr & $\mathrm{Lb}$ & & 420 & 2470 & 3 & 7.4 & 2.1 & & 1.3 & 2 \\
\hline U Men & $\mathrm{SRa}$ & 407 & 320 & 2160 & 2.0 & 7.2 & 1.7 & & & 1 \\
\hline T Mic & $\mathrm{SRb}$ & 347 & 130 & 2430 & 0.8 & 4.8 & 1.2 & & 1.6 & 2 \\
\hline EX Ori & $\mathrm{Lb}$ & & 470 & 2490 & 0.8 & 4.2 & 1.3 & & 2.2 & 2 \\
\hline V352 Ori & $\mathrm{Lb}$ & & 250 & 2560 & 0.5 & 8.4 & 0.9 & & & 1 \\
\hline S Pav & $\mathrm{SRa}$ & 381 & 150 & 2190 & 0.8 & 9.0 & 1.1 & & & 1 \\
\hline SV Peg & $\mathrm{SRb}$ & 145 & 190 & 2330 & 3 & 7.5 & 2.1 & 0.1 & 4.2 & 3 \\
\hline TW Peg & $\mathrm{SRb}$ & 929 & 200 & 2690 & 2.5 & 9.5 & 1.8 & 0.25 & 3.5 & 4 \\
\hline V PsA & $\mathrm{SRb}$ & 148 & 220 & 2360 & 3 & 14.4 & 1.9 & & & 1 \\
\hline $\mathrm{L}^{2}$ Pup & $\mathrm{SRb}$ & 141 & 85 & 2690 & 0.2 & 2.3 & 0.7 & 0.05 & 0.9 & 4 \\
\hline OT Pup & $\mathrm{Lb}$ & & 500 & 2630 & 5 & 9.0 & 2.6 & & 4.8 & 2 \\
\hline Y Scl & $\mathrm{SRb}$ & & 330 & 2620 & 1.3 & 5.2 & 1.5 & & 2.0 & 2 \\
\hline CZ Ser & $\mathrm{Lb}$ & & 440 & 2150 & 8 & 9.5 & 3.2 & & 0.2 & 2 \\
\hline$\tau^{4} \operatorname{Ser}$ & $\mathrm{SRb}$ & 100 & 170 & 2500 & 1.5 & 14.4 & 1.5 & & & 1 \\
\hline SU Sgr & $\mathrm{SRb}$ & 60 & 240 & 2090 & 4 & 9.5 & 2.3 & & 4.7 & 2 \\
\hline UX Sgr & $\mathrm{SRb}$ & 100 & 310 & 2520 & 1.5 & 9.5 & 1.5 & & & 1 \\
\hline V1943 Sgr & $\mathrm{Lb}$ & & 150 & 2250 & 1.3 & 5.4 & 1.4 & 0.5 & 9.2 & 3 \\
\hline V Tel & $\mathrm{SRb}$ & 125 & 290 & 2260 & 2.0 & 6.8 & 1.6 & & 9.5 & 2 \\
\hline
\end{tabular}

\footnotetext{
${ }^{1}$ Distance derived assuming a luminosity of $4000 L_{\odot}$.
} 
Table 3. continued.

\begin{tabular}{|c|c|c|c|c|c|c|c|c|c|c|}
\hline Source & $\begin{array}{l}\text { Var. } \\
\text { type }\end{array}$ & $\begin{array}{c}P \\
\text { [days] }\end{array}$ & $\begin{array}{r}D^{1} \\
{[\mathrm{pc}]}\end{array}$ & $\begin{array}{l}T_{\mathrm{bb}} \\
{[\mathrm{K}]}\end{array}$ & $\begin{array}{c}\dot{M} \\
{\left[10^{-7} M_{\odot} \mathrm{yr}^{-1}\right]}\end{array}$ & $\begin{array}{c}v_{\mathrm{e}} \\
{\left[\mathrm{km} \mathrm{s}^{-1}\right]}\end{array}$ & $\begin{array}{c}r_{\mathrm{p}} \\
{\left[10^{16} \mathrm{~cm}\right]}\end{array}$ & $h$ & $\chi_{\mathrm{red}}^{2}$ & $\mathrm{~N}$ \\
\hline Y Tel & $\mathrm{Lb}$ & & 340 & 2350 & 0.5 & 3.5 & 1.0 & & 13.3 & 2 \\
\hline AZ UMa & $\mathrm{Lb}$ & & 490 & 2620 & 2.5 & 4.5 & 2.1 & & 2.7 & 2 \\
\hline Y UMa & $\mathrm{SRb}$ & 168 & 220 & 2230 & 1.5 & 4.8 & 1.7 & 0.5 & 0.9 & 3 \\
\hline SU Vel & $\mathrm{SRb}$ & 150 & 250 & 2380 & 2.0 & 5.5 & 1.8 & 0.2 & 2.9 & 3 \\
\hline BK Vir & $\mathrm{SRb}$ & 150 & 190 & 2210 & 1.5 & 4.0 & 1.9 & 0.05 & 0.1 & 3 \\
\hline RT Vir & $\mathrm{SRb}$ & 155 & 170 & 2110 & 5 & 7.8 & 2.7 & 0.05 & 0.5 & 4 \\
\hline RW Vir & $\mathrm{Lb}$ & & 280 & 2530 & 1.5 & 7.0 & 1.5 & & 1.1 & 2 \\
\hline SW Vir & $\mathrm{SRb}$ & 150 & 120 & 2190 & 4 & 7.5 & 2.2 & 0.1 & 0.7 & 6 \\
\hline
\end{tabular}

${ }^{1}$ Distance derived assuming a luminosity of $4000 L_{\odot}$.
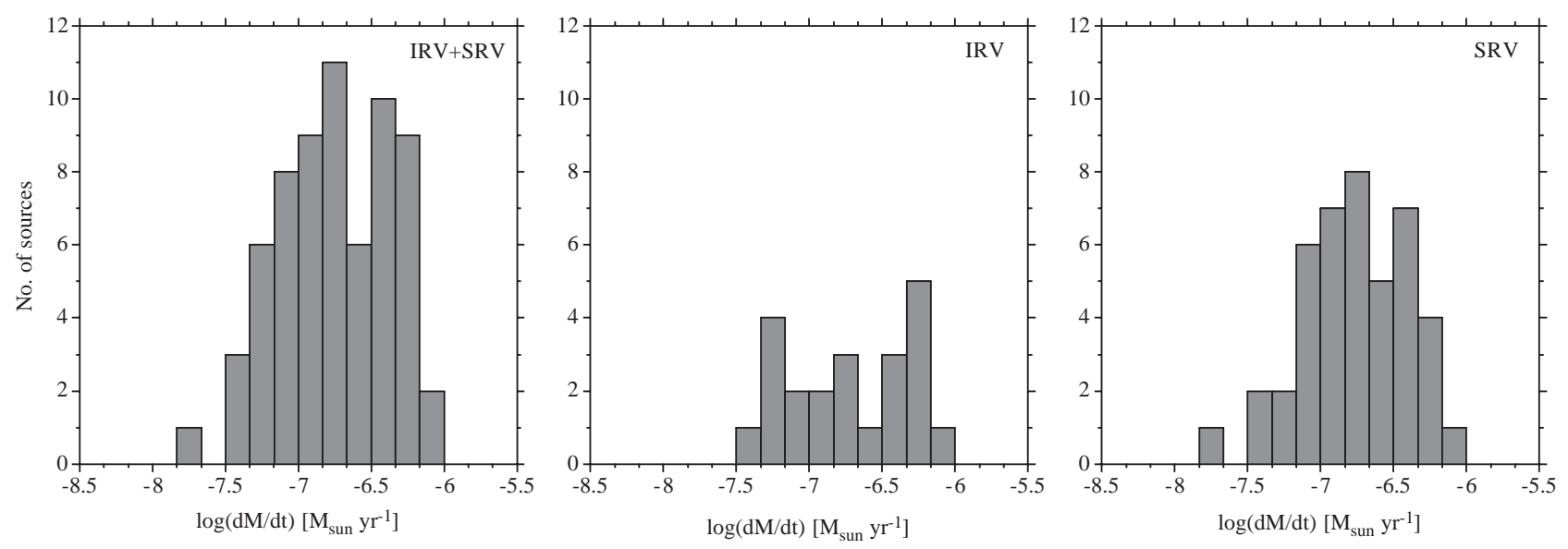

Fig. 4. Mass loss rate distribution for the whole sample (left panel), as well as for the IRVs (middle) and SRVs (right) separately. The objects with double-component line profiles are excluded.
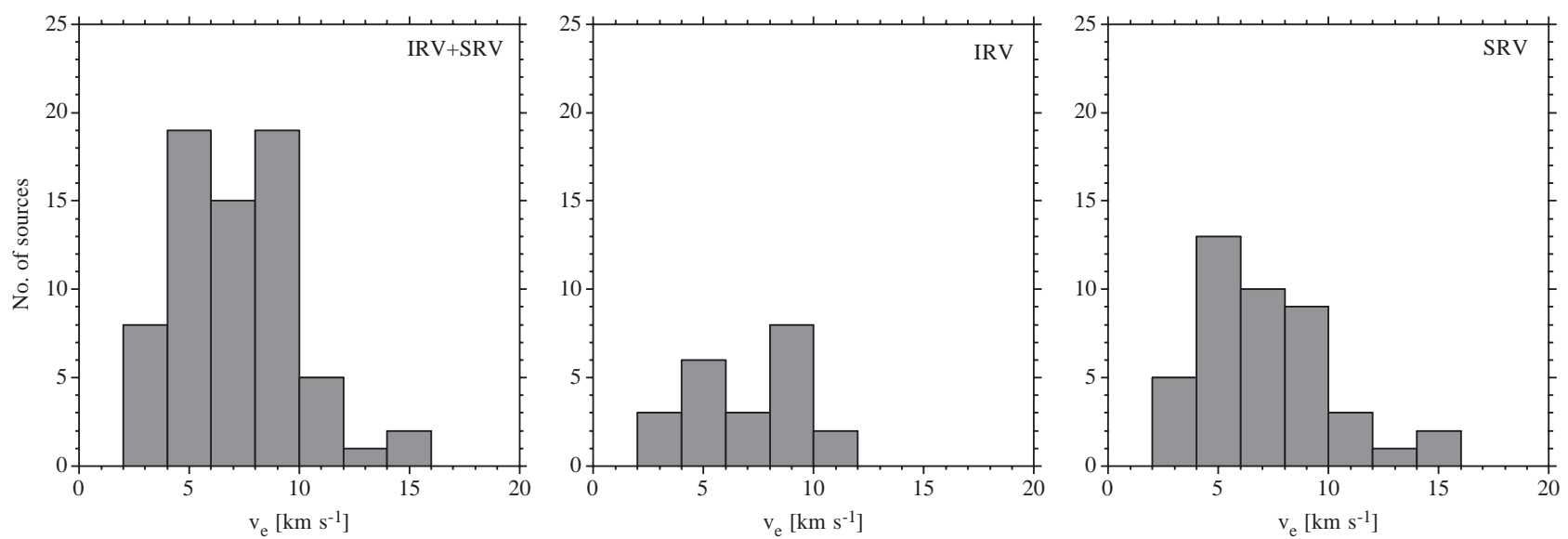

Fig. 5. Gas expansion velocity distribution for the whole sample (left panel), as well as for the IRVs (middle) and SRVs (right) separately. The objects with double-component line profiles are excluded.

\subsection{The mass loss rate distribution}

The distribution of the derived mass loss rates have a median value of $2.0 \times 10^{-7} M_{\odot} \mathrm{yr}^{-1}$, and a minimum of $2.0 \times$ $10^{-8} M_{\odot} \mathrm{yr}^{-1}$ and a maximum of $8 \times 10^{-7} M_{\odot} \mathrm{yr}^{-1}$. There is no significant difference between the IRVs and the SRVs, but the number of IRVs is quite low. We believe that these mass loss rate distributions are representative for the mass losing M-type IRVs and SRVs on the AGB (see arguments below).
We find no significant difference when comparing with the sample of C-type IRVs and SRVs, where the median was $1.6 \times 10^{-7} M_{\odot} \mathrm{yr}^{-1}$. Therefore, the mass loss rates of these types of variables appear independent of chemistry (also for the $\mathrm{C}$-stars there is no dependence on the $\mathrm{C} / \mathrm{O}$-ratio). This conclusion rests on the uncertain assumptions of the $\mathrm{CO}$ fractional abundance $\left(10^{-3}\right.$ in the case of the C-star sample, as opposed to the value $2 \times 10^{-4}$ used here for the M-stars). 
Most notable is the sharp cut-off at a mass loss rate slightly below $10^{-6} M_{\odot} \mathrm{yr}^{-1}$. This is very likely not a selection effect. All our stars are included in the GCVS, and even though this favours less obscured stars the GCVS contains many stars with mass loss rates in excess of this value. Thus, there appears to be an upper limit for the mass loss rate of an M-type IRV or SRV on the AGB. Regular pulsators of M-type, Miras and $\mathrm{OH} / \mathrm{IR}$-stars, clearly reach significantly higher mass loss rates, and hence the regularity of the pulsation and its amplitude play an important role for the magnitude of the mass loss rate. Some caution must be exercised here though. We are averaging the mass loss rate over a time scale of about one thousand years, and there are indications that the mass loss rates of IRV/SRVs are more time-variable, on shorter time scales than this, than are the mass loss rates of the Miras (Marengo et al. 2001). This would lead to an, on the average, lowered mass loss rate of an IRV/SRV.

The decrease in the number of objects with low mass loss rates could indicate an effect of limited observational sensitivity. However, a plot of the mass loss rate as a function of the distance suggests that this is not the case, Fig. 6. We detect low mass loss rate objects out to about $500 \mathrm{pc}$, and beyond this only a few higher mass loss rate objects are detected. That is, nearby stars with mass loss rates below $10^{-8} M_{\odot} \mathrm{yr}^{-1}$ should be detectable. (We checked all the non-detections reported by Kerschbaum \& Olofsson 1999, and found that in no case do they provide an upper limit in the mass loss rate which is significantly lower than a few $10^{-8} M_{\odot} \mathrm{yr}^{-1}$.) Hence, we interpret the trailing off at low mass loss rates as due to a lack of such sources among the mass losing M-type IRVs and SRVs on the AGB. However, our sample is limited by the IRAS colour [12]-[25] (Sect. 2). Therefore, it is possible that there exists M-type AGB-stars with mass loss rates lower than our limit of about $10^{-8} M_{\odot} \mathrm{yr}^{-1}$. The case for the C-stars is different. Schöier \& Olofsson (2001) also derived a lower limit of about $10^{-8} M_{\odot} \mathrm{yr}^{-1}$, but this is based on a $K$-magnitude limited sample, for which the $K$-magnitude is expected to be relatively constant, where all stars within about $500 \mathrm{pc}$ were detected (Olofsson et al. 1993).

We have also compared the derived mass loss rates with the periods of the SRVs, Fig. 7. The conclusion by Kerschbaum et al. (1996) that for these objects the period of pulsation plays no role for the mass loss rate still holds. The apparent absence of stars with periods in the range 200-300 days is most probably due to a distinct division into two pulsational modes, one operating only below 200 days and one only above 300 days. Likewise, for the C-SRVs we find no correlation between mass loss rate and period (the gap between 200 and 300 days does not exist for these stars).

\subsection{Mass loss and stellar temperature}

It has turned out to be very difficult to derive the mass loss rate of an AGB-star from first principles. Some attempts have nevertheless been made and they all indicate a strong dependence on the stellar effective temperature, due to its effect on grain condensation (Arndt et al. 1997; Winters et al. 2000).

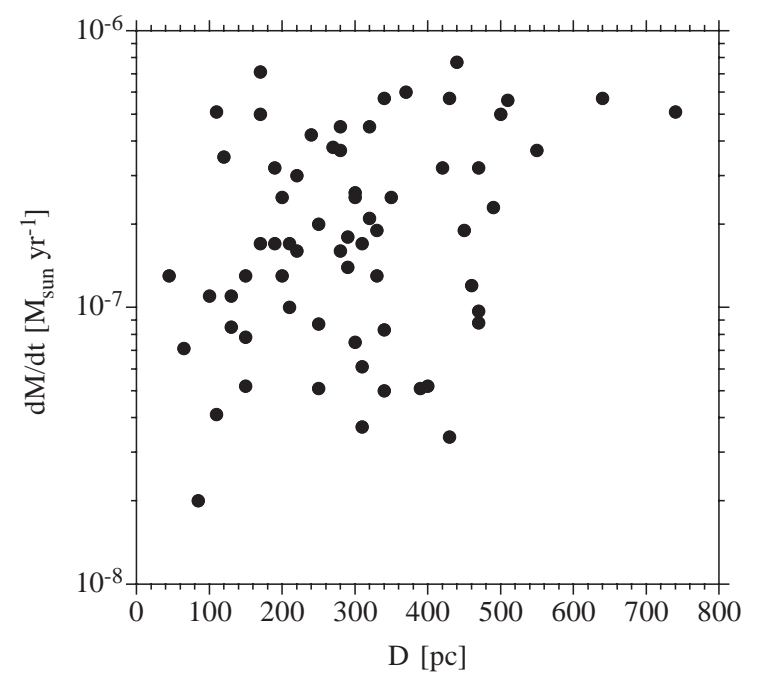

Fig. 6. The derived mass loss rate as a function of the distance to the object.

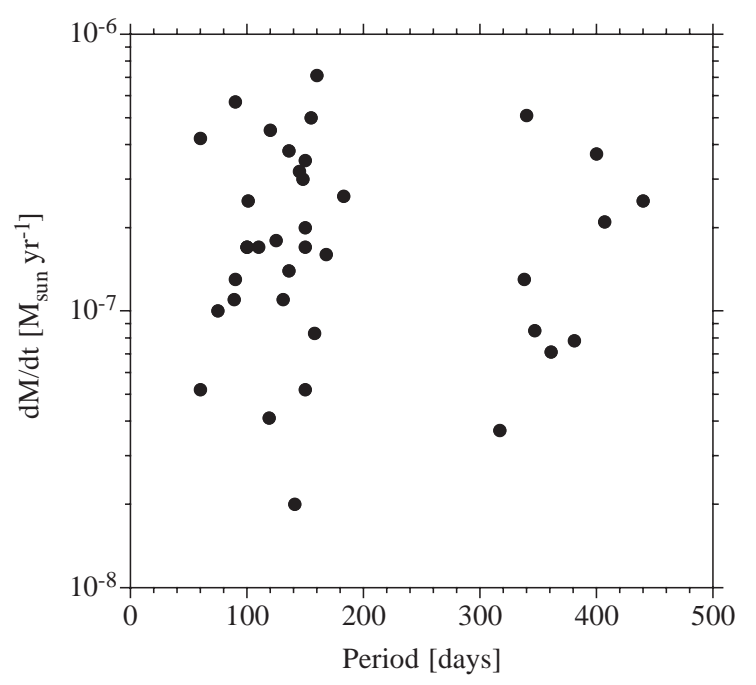

Fig. 7. The derived mass loss rate as a function of the period of pulsation for the SRVs.

The blackbody temperatures derived for our stars are at least indicative of the stellar effective temperatures, even though there may be a systematic effect (Sect. 4.2.4). Of course, a high mass loss rate will lead to significant dust emission and this may have an effect on the stellar blackbody temperature estimate, but the approach with two blackbodies should to some extent diminish this effect. We have in Fig. 8 plotted the derived mass loss rate as a function of the stellar blackbody temperature. Clearly, there is no correlation at all. Even taking into account the somewhat uncertain relation between our stellar blackbody temperature and the effective temperature this shows that for these objects with relatively low mass loss rates the temperature in the stellar atmosphere plays no role. The C-type IRVs and SRVs show the same absence of a correlation, only when the C-Miras are added is there a weak trend with mass loss rate decreasing with increasing temperature. 


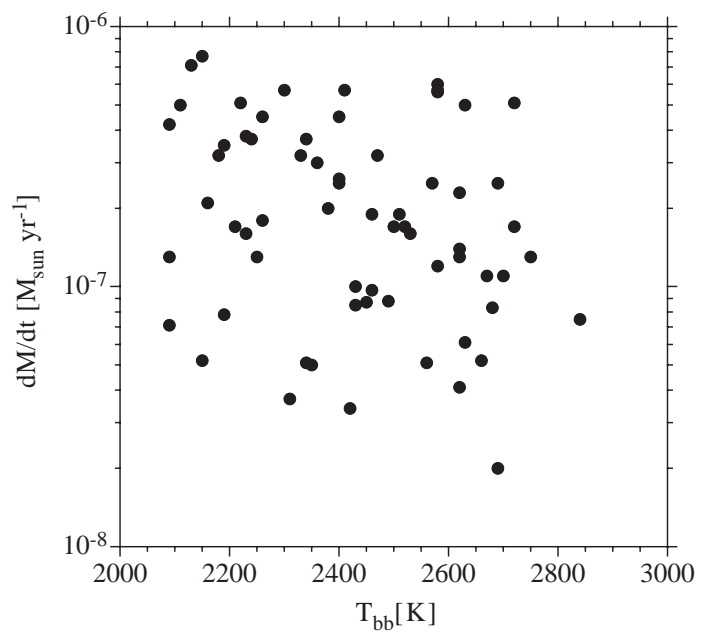

Fig. 8. The derived mass loss rate as a function of the stellar blackbody temperature.

\subsection{The gas expansion velocity distribution}

The gas expansion velocities derived from the model fits have a distribution with a median for the whole sample of $7.0 \mathrm{~km} \mathrm{~s}^{-1}$, and a minimum of $2.2 \mathrm{~km} \mathrm{~s}^{-1}$ and a maximum of $14.4 \mathrm{~km} \mathrm{~s}^{-1}$, i.e., clearly these objects sample the low gas expansion velocity end of AGB winds. Similar results have been obtained for short-period M-Miras (Young 1995; Groenewegen et al. 1999). We find no apparent difference between the IRV and the SRV distributions, but the former is based on relatively few objects. A comparison with the C-type IRVs and SRVs, for which the median is $9.5 \mathrm{~km} \mathrm{~s}^{-1}$ ( 39 objects) and where the fraction of lowvelocity sources is much lower, indicates that in this respect there is a difference between the chemistries. A C-type chemistry produces higher gas expansion velocities. The large fraction of low-velocity sources in our sample is further discussed in Sect 6.5.

\subsection{Mass loss and envelope kinematics}

There are two main characteristics of the mass loss process, the stellar mass loss rate and the circumstellar gas expansion velocity. The former is to a large extent determined by the conditions at the transonic point, i.e., the density at the point where the gas velocity goes from being sub- to supersonic, while the latter is determined by the acceleration beyond this point. Hence, these two properties do not necessarily correlate with each other. However, in a study of a dust-driven wind Habing et al. (1994) found that the relation $\dot{M} \propto v_{\mathrm{e}}$ should apply in the optically thin limit. Solving the same set of equations Elitzur \& Ivezic (2001) found that the dependence becomes even stronger, $\dot{M} \propto v_{\mathrm{e}}^{3}$, when the effect of gravity is negligible. Therefore, a comparison between the two mass loss characteristics may provide important results, which any mass loss mechanism model must be able to explain.

In Fig. 9 we present the mass loss rates and gas expansion velocities for our sample. There is definitely a trend in the sense that the mass loss rate and gas expansion velocity increase jointly. A linear fit to the data results in $\dot{M} \propto v_{\mathrm{e}}^{1.4}$ with

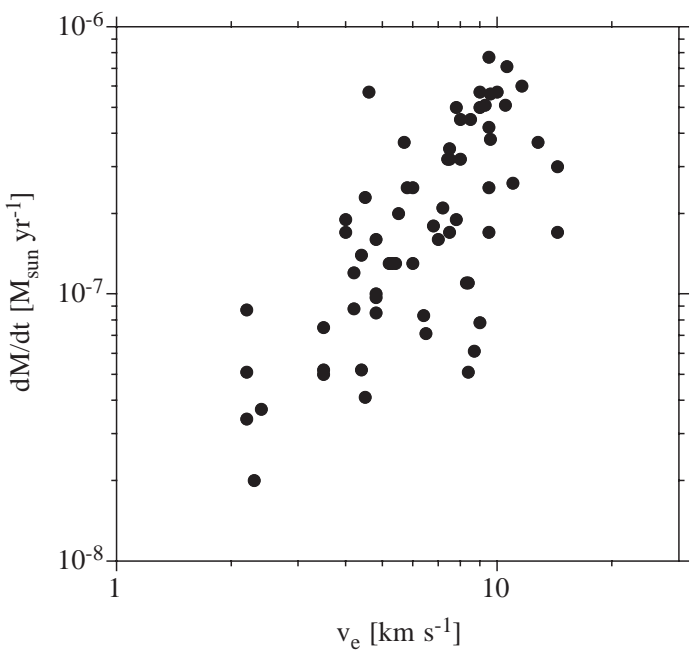

Fig. 9. The derived mass loss rate as a function of the gas expansion velocity for the whole sample, excluding the double-component line profiles.

a correlation coefficient of 0.68 . For the $\mathrm{C}$-star IRVs and SRVs the corresponding result is $\dot{M} \propto v_{\mathrm{e}}^{2.0}$ with a correlation coefficient of 0.76 . Thus, the dependence is weaker for the M-stars, but this result is hardly significant. The spread in mass loss rate for a given velocity is substantial, and larger than the estimated uncertainty in the mass loss rate. Results of similar nature have been found for other samples of stars. Young (1995) found a strong dependence, $\dot{M} \propto v_{\mathrm{e}}^{3.4}$, for a sample of short-period MMiras, and Knapp et al. (1998) found $\dot{M} \propto v_{\mathrm{e}}^{2}$ for a sample containing a mixture of $\mathrm{M}$ - and C-stars. Differences in the slope may occur if different methods for estimating mass loss rates have systematic trends, e.g., a systematic underestimate at low mass loss rates and low expansion velocities, but also the range of mass loss rates covered, the types of variables, etc., will have an effect.

Nevertheless, we can conclude that the mass loss mechanism(s) produces a correlation between its two main characteristics which is in line with theoretical predictions for an optically thin, dust-driven wind. Our result is also consistent with a rather weak dependence for low luminosity sources where gravity effects cannot be ignored. The considerable spread in mass loss rate for a given velocity suggests that the outcome for an individual star is sensitive to the conditions in the region where these properties are determined.

\subsection{Low gas expansion velocity sources}

The fraction of objects with low gas expansion velocities is high in our sample. There are 20 objects (i.e., $30 \%$ of the whole sample) with velocities lower than $5 \mathrm{~km} \mathrm{~s}^{-1}$. The corresponding value among the carbon star IRVs and SRVs is only $8 \%$. There is no sample selection bias for or against low velocity sources in any of the samples, and since the detection rates are high for both samples, we conclude that there is clearly a difference between the mass loss properties of AGB-stars of M- and C-type in this respect. 

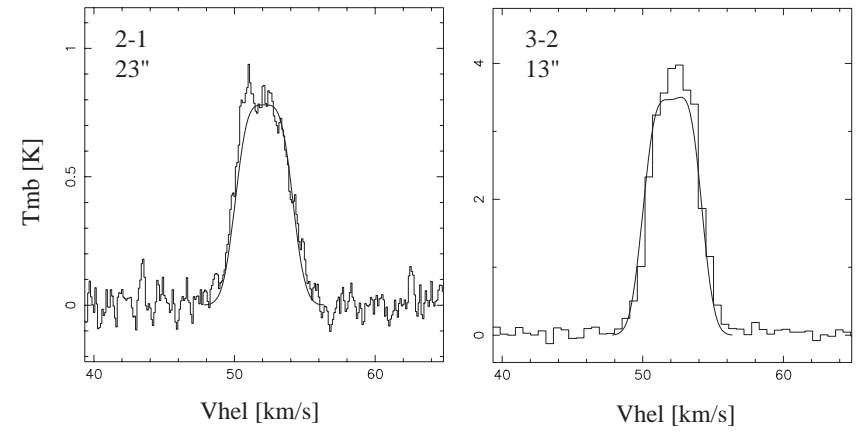

Fig. 10. $\mathrm{CO}(J=2 \rightarrow 1)$ and $\mathrm{CO}(J=3 \rightarrow 2)$ spectra (histograms) obtained at the SEST and the JCMT, respectively, and model line profiles (thin, solid lines) for the low gas expansion velocity source $\mathrm{L}^{2}$ Pup (the beam size is given in each panel).

Of particular interest for further studies are the sources with gas expansion velocities lower than $3 \mathrm{~km} \mathrm{~s}^{-1}$ : V584 Aql, T Ari, BI Car, RX Lac, and $\mathrm{L}^{2}$ Pup. Such a low velocity corresponds to the escape velocity at a distance of $1.5 \times 10^{15} \mathrm{~cm}$ for a $1 M_{\odot}$ star, i.e., a distance corresponding to about 100 stellar radii, considerably larger than the normally accepted acceleration zone, about 20 stellar radii (Habing et al. 1994). In a detailed study of state of the art mass loss models Winters et al. (2000) concluded that for low radiative acceleration efficiencies only low mass loss rates $\left(\lesssim 3 \times 10^{-7} M_{\odot} \mathrm{yr}^{-1}\right)$ and low gas expansion velocities $\left(\lesssim 5 \mathrm{~km} \mathrm{~s}^{-1}\right)$ are produced (their class B models). In these models the gas expands at a relatively constant and low velocity beyond a few stellar radii, and it finally exceeds the escape velocity at large radii. This can provide an explanation for the low velocity sources, but also more complicated geometries/kinematics may play an important role (Sect. 6.7).

Jura et al. (2002) presented a study of $\mathrm{L}^{2}$ Pup where they used the mid-IR cameras on the Keck telescope to partly resolve the dust emission. No clear geometrical structure is evident, but they derive a dust mass loss rate of $10^{-9} M_{\odot} \mathrm{yr}^{-1}$, which combined with our gas mass loss rate, results in a dustto-gas mass ratio as high as 0.05 , i.e., about 15 times higher than our average estimate from the $h$-parameter (Sect. 5.2). This suggests that there are problems with the dust and/or the gas mass loss rate estimates of this star. In Fig. 10 we show the model line profiles superimposed on our highest quality spectra of this object. A very good fit is obtained for a mass loss rate of $2.2 \times 10^{-8} M_{\odot} \mathrm{yr}^{-1}$, an expansion velocity of $2.1 \mathrm{~km} \mathrm{~s}^{-1}$, and a turbulent velocity width of $1.0 \mathrm{~km} \mathrm{~s}^{-1}$ (this is higher than the $0.5 \mathrm{~km} \mathrm{~s}^{-1}$ adopted in the modelling of the whole sample). However, this object is so extreme that not too much faith should be put in the model results despite the successful fit.

\subsection{The line profiles}

The line profiles carry information which was not used to constrain the derived mass loss rates. In this section we briefly discuss how well the model line profiles reproduce the observed ones. In general, the results are satisfactory. However, although there are both too double-peaked and too rounded model profiles when compared with the observed ones, there is a trend of too double-peaked (or too flat) model line profiles, indeed observed double-peaked line profiles are very rare. The discrepancy is worst for the $J=1 \rightarrow 0$ line, where double-peaked model line profiles are common. There are two possibilities for such a discrepancy, either the angular size of the emitting region is too large in the model or there is an effect due to maser emission, which is not reproduced in nature. The former can be due to systematically too small distances to the sources or too large photodissociation radii. The latter is a possibility because for these low mass loss rate objects radiative excitation plays an important role and it tends to invert preferentially the lower $J$-transitions. However, among the objects with discrepancies there are about as many without maser action as with maser action (in the model).

The by far worst discrepancy is obtained for R Dor, where all the model line profiles are strongly double-peaked, Fig. 11. All three transitions are inverted (the $J=1 \rightarrow 0$ line over the whole radial range, but the $J=2 \rightarrow 1$ and $J=3 \rightarrow 2$ lines only over a part of it), but the optical depths are so low that substantial effects of maser action are not expected. The double-peakedness is rather due to the large angular extent of the emission in the model, i.e., the emission is clearly spatially resolved. This can be solved by increasing the distance. The somewhat uncertain Hipparcos distance of R Dor is $61 \mathrm{pc}$, but a change to this distance leads only to marginal improvements in the model fitting. We have to increase the distance by a factor of three to get acceptable fits to the observed data $(D=150 \mathrm{pc}$ for which the best-fit results are $\dot{M}=5 \times 10^{-7} M_{\odot} \mathrm{yr}^{-1}$ and $h=1.5$; note that the derived temperature structure depends on the distance to the source due to the emission being spatially resolved). Such a large distance is not obviously compatible with the Hipparcos data. Alternatively, we can artificially lower the size of the $\mathrm{CO}$ envelope by a factor of three compared to that given by the model of Mamon et al. (1988) (for which the best fit results are $\dot{M}=3 \times 10^{-7} M_{\odot} \mathrm{yr}^{-1}$ and $h=0.05$; for this mass loss rate the photodissociation radius is actually five times larger according to the model of Mamon et al. 1988). Actual tests of the predictions of the model of Mamon et al. (1988) have mainly been done for high mass loss rate C-stars (Schöier \& Olofsson 2000, 2001). The model has passed these tests, and it is therefore questionable whether it gives results off by a factor of five at lower mass loss rates. A possibility exist that the interstellar UV field is exceptionally strong and hence limits severely the $\mathrm{CO}$ envelope of $\mathrm{R}$ Dor. Alternatively, the mass loss is highly variable, and the small outer radius of the $\mathrm{CO}$ envelope is a consequence of a recent higher mass loss epoch. We must conclude that presently the reason for the major discrepancy between the observational data of R Dor and our modelling results is not clear. A complicating factor is that the $J=1 \rightarrow 0$ line profile is time variable (Nyman, priv. comm.), and that it at times looks rather peculiar (compare e.g. the spectrum shown in Lindqvist et al. 1992).

Finally, we note here that Olofsson et al. (1993) in their sample of C-type SRVs and IRVs found five sources (about $10 \%$ ) with remarkable $\mathrm{CO}$ radio line profiles, sharply doublepeaked with only very weak emission at the systemic velocity. These were subsequently shown to originate in large, detached shells (Olofsson et al. 1996), which are geometrically very thin (Lindqvist et al. 1999; Olofsson et al. 2000), presumably an 

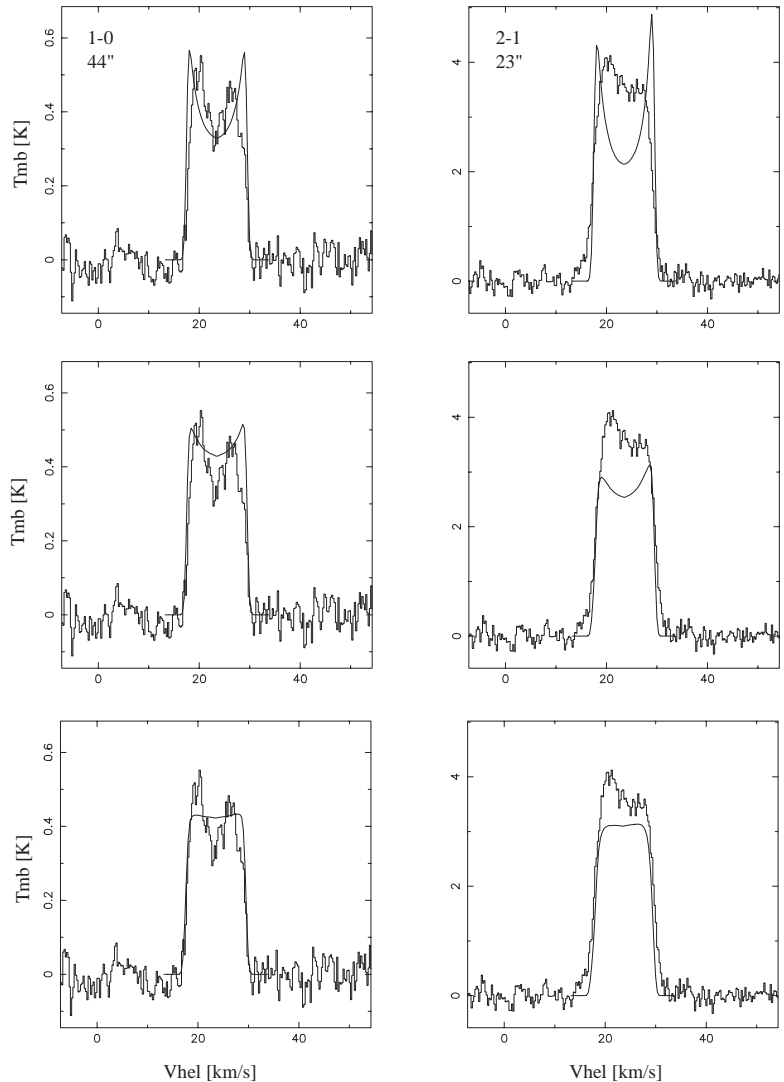

effect of highly episodic mass loss. We have found no such source in our sample of M-stars. Hence, in this respect there must be a difference in the mass loss properties between the two chemistries.

\subsection{Double-component line profiles}

Knapp et al. (1998) and Kerschbaum \& Olofsson (1999) were the first to discuss more thoroughly the small number of objects with line profiles which can be clearly divided into two components, a narrow feature centred on a broader plateau. Such sources exist both among M- and C-type stars (Knapp et al. 1998). The origin of such a line profile is still not clear. Knapp et al. (1998) argued that it is an effect of episodic mass loss with highly varying gas expansion velocities. Alternatively, it can be an effect of complicated geometries/kinematics. The first spatial information was provided by Kahane \& Jura (1996). A CO radio line map towards the M-star X Her suggested that the broad plateau is a bipolar outflow, while the narrow feature was not spatially resolved. Bergman et al. (2000) produced CO radio line interferometry maps of the M-star RV Boo. In this case the brightness distributions suggest that the broad plateau emission comes from a circumstellar disk with Keplerian rotation. Kahane et al. (1998) and Jura \& Kahane (1999) interpret the narrow $\mathrm{CO}$ radio line features which they observe in a few cases as originating in reservoirs of orbiting gas (these sources do not have distinct double-component line profiles). It is fair to say that no consensus has been reached about these peculiar circumstellar emissions.
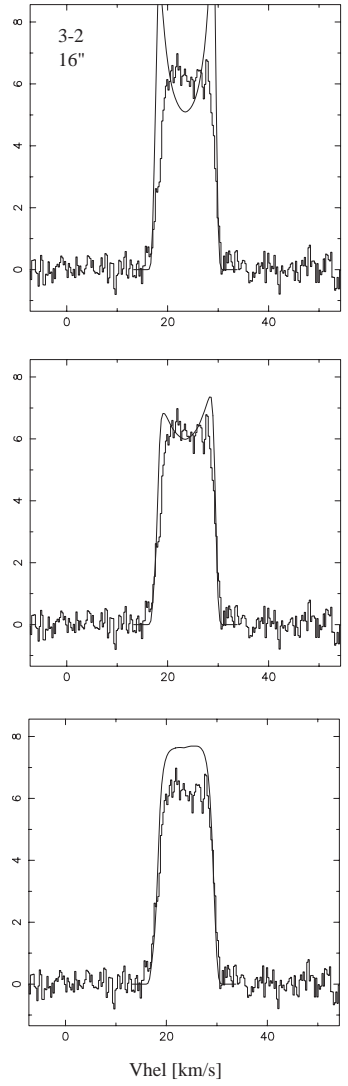

Fig. 11. Observed and modelled line profiles for R Dor. Upper panels show the results of the nominal model of $\mathrm{R}$ Dor $(D=$ $\left.46 \mathrm{pc}, \dot{M}=1.3 \times 10^{-7} M_{\odot} \mathrm{yr}^{-1}, h=0.7\right)$. The middle panels show the results for a distance three times larger $(D=150 \mathrm{pc}$, $\left.\dot{M}=5 \times 10^{-7} M_{\odot} \mathrm{yr}^{-1}, h=1.5\right)$. The lower panels show the results for a photodissociation radius three times smaller than that of the nominal model $\left(\dot{M}=3 \times 10^{-7} M_{\odot} \mathrm{yr}^{-1}\right.$, $h=0.05)$.

In our sample we have four sources of this type, EP Aqr, RV Boo, X Her, and SV Psc, all of them SRVs. We have simply decomposed the emission into two components for each source, assuming that the emissions are additive. Mass loss rates and gas expansion velocities were determined in the same way as for the rest of our objects. This is probably a highly questionable approach for both components. The results, as well as some source information, are given in Table 4. Knapp et al. (1998) derived mass loss rates for EP Aqr and X Her which are within a factor of two of our estimates (both for the narrow and the broad components).

Not unexpectedly the mass loss rates are higher for the broader component by, on average, an order of magnitude. The fits to the narrow components result in very low gas expansion velocities. Indeed, so low, e.g., $1.0 \mathrm{~km} \mathrm{~s}^{-1}$ in the case of EP Aqr, that an interpretation in the form of a spherical outflow is put to question. On the other hand, the relation between mass loss rate and gas expansion velocity is the same for these objects as for the rest of the sources, Fig. 9. The narrow component gas appears cooler than the broad emission gas in those three cases where an $h$ can be estimated. This may be an accidental result, but it can also provide a clue to the interpretation.

\section{Conclusions}

We have determined mass loss rates and gas expansion velocities for a sample of 69 M-type IRVs (22 objects) and SRVs (47 objects) on the AGB using a radiative transfer code to model their circumstellar CO radio line emission. We believe that this sample is representative for the mass losing stars of 
Table 4. Source parameters and model results for those objects with double component line profiles.

\begin{tabular}{|c|c|c|c|c|c|c|c|c|c|c|c|}
\hline Source & $\begin{array}{l}\text { Var. } \\
\text { type }\end{array}$ & $\begin{array}{c}P \\
\text { [days] }\end{array}$ & $\begin{array}{r}D \\
{[\mathrm{pc}]}\end{array}$ & $\begin{array}{l}T_{\mathrm{bb}} \\
{[\mathrm{K}]}\end{array}$ & comp. & $\begin{array}{c}\dot{M} \\
{\left[10^{-7} M_{\odot} \mathrm{yr}^{-1}\right]}\end{array}$ & $\begin{array}{c}v_{\mathrm{e}} \\
{\left[\mathrm{km} \mathrm{s}^{-1}\right]}\end{array}$ & $\begin{array}{c}r_{\mathrm{p}} \\
{\left[10^{16} \mathrm{~cm}\right]}\end{array}$ & $h$ & $\chi_{\text {red }}^{2}$ & $N$ \\
\hline \multirow[t]{2}{*}{ EP Aqr } & $\mathrm{SRb}$ & 55 & 140 & 2200 & broad & 5 & 9.2 & 2.5 & & & 1 \\
\hline & & & & & narrow & 0.3 & 1.0 & 1.1 & & & 1 \\
\hline \multirow[t]{2}{*}{ RV Boo } & $\mathrm{SRb}$ & 137 & 280 & 2760 & broad & 2.0 & 7.0 & 1.8 & 0.1 & 0.7 & 4 \\
\hline & & & & & narrow & 0.3 & 2.3 & 0.8 & 0.05 & 9.9 & 4 \\
\hline \multirow[t]{2}{*}{ X Her } & $\mathrm{SRb}$ & 95 & 140 & 2490 & broad & 1.5 & 6.5 & 1.5 & 0.2 & 4.6 & 3 \\
\hline & & & & & narrow & 0.4 & 2.2 & 1.0 & 0.03 & 4.5 & 3 \\
\hline \multirow[t]{2}{*}{ SV Psc } & $\mathrm{SRb}$ & 102 & 380 & 2450 & broad & 3 & 9.5 & 1.9 & 0.1 & 2.1 & 3 \\
\hline & & & & & narrow & 0.3 & 1.5 & 1.0 & 0.05 & 20.7 & 3 \\
\hline
\end{tabular}

this type. The uncertainties in the estimated mass loss rates are rather low within the adopted stellar/circumstellar model, typically less than $\pm 50 \%$. However, a sensitivity analysis shows that for these low mass loss rate stars there is a considerably uncertainty due to the stellar luminosity, the size of the CO envelope, the $\mathrm{CO}$ abundance, and as usual the distance to the source. We find that the mass loss rates determined by the detailed radiative transfer analysis differ by almost an order of magnitude from those obtained by published mass loss rate formulae.

The (molecular hydrogen) mass loss rate distribution has a median value of $2.0 \times 10^{-7} M_{\odot} \mathrm{yr}^{-1}$, and a minimum of $2.0 \times 10^{-8} M_{\odot} \mathrm{yr}^{-1}$ and a maximum of $8 \times 10^{-7} M_{\odot} \mathrm{yr}^{-1}$. M-type IRVs and SRVs with a mass loss rate in excess of $5 \times$ $10^{-7} M_{\odot} \mathrm{yr}^{-1}$ must be very rare, and in this respect the regularity and amplitude of the pulsation plays an important role. We also find that among these mass losing stars the number of sources with mass loss rates below a few $10^{-8} M_{\odot} \mathrm{yr}^{-1}$ must be small.

We find no significant difference between the IRVs and the SRVs in terms of their mass loss characteristics. Among the SRVs the mass loss rate shows no dependence on the period. Thus, for these non-regular, low-amplitude pulsators it appears that the pulsational pattern plays no role for the mass loss efficiency.

We have determined temperatures for our sample stars by fitting blackbody curves to their spectral energy distributions. These blackbody temperatures have been shown to correlate reasonably well with the stellar effective temperatures. The mass loss rates of our stars show no correlation at all with these stellar blackbody temperatures.

The gas expansion velocity distribution has a median of $7.0 \mathrm{~km} \mathrm{~s}^{-1}$, and a minimum of $2.2 \mathrm{~km} \mathrm{~s}^{-1}$ and a maximum of $14.4 \mathrm{~km} \mathrm{~s}^{-1}$. No doubt, these objects sample the low gas expansion velocity end of AGB winds. The fraction of objects with low gas expansion velocities is high, about $30 \%$ have velocities lower than $5 \mathrm{~km} \mathrm{~s}^{-1}$. There are four objects with gas expansion velocities lower than $3 \mathrm{~km} \mathrm{~s}^{-1}$ : V584 Aql, T Ari, BI Car, RX Lac, and $\mathrm{L}^{2}$ Pup. These objects certainly deserve further study.

We find that the mass loss rate and the gas expansion velocity correlate well, $\dot{M} \propto v_{\mathrm{e}}^{1.4}$, even though for a given velocity (which is well determined) the mass loss rate may take on a value within a range of a factor of five (the uncertainty in the mass loss rate estimate is lower than this within the adopted circumstellar model). The result is in line with theoretical predictions for an optically thin, dust-driven wind.

A more detailed test of the $\mathrm{CO}$ modelling is provided by the shape of the line profiles. In general, the fits are acceptable, but there is a trend that the model profiles, in particular the $J=1 \rightarrow 0$ ones, are more flat-topped, or even weakly doublepeaked, than the observed ones. An exceptional case is R Dor, where the high-quality, observed line profiles are essentially flat-topped, while the model ones are sharply double-peaked. Acceptable fits are obtained by increasing the distance to the star or by artificially decreasing the size of the CO envelope.

The sample contains four sources with distinctly doublecomponent CO line profiles: EP Aqr, RV Boo, X Her, and SV Psc (all SRVs). We have modelled the two components separately for each star and derive mass loss rates and gas expansion velocities using the same circumstellar model as for the rest of the sample. The resulting mass loss rates and gas expansion velocities show the same positive correlation as that of the other objects. At present, the exact nature(s) of these objects is unknown.

We have compared the results of this M-star sample with a similar C-star sample. The mass loss rate distributions are comparable, suggesting no dependence on chemistry for these types of objects. Likewise, the mass loss rates of the C-stars show no correlation with stellar temperature or period. The gas expansion velocity distributions though are clearly different. The fraction of low velocity sources is much higher in the M-star sample. In both cases there is a correlation between mass loss rate and gas expansion velocity, although the detailed relations are different. Our crude estimates of the dust properties, through the gas-grain collision heating term, indicate that the two samples have similar gas-to-dust ratios and that these differ significantly from that of high mass loss rate Cstars. This also means that the gas-CSEs due to low mass loss rates are cooler than expected from a simple extrapolation of the results for IRC +10216 . Finally, we find no example of the sharply double-peaked $\mathrm{CO}$ line profile, which is evidence of a large, detached CO-shell, among the M-stars. About 10\% of the C-stars show this phenomenon.

Acknowledgements. We are grateful to the referee, M. Marengo, for a careful reading of the manuscript and for constructive comments. We also thank L.- $\AA$. Nyman for giving us the $\mathrm{R} \operatorname{Dor} \operatorname{CO}(J=1 \rightarrow 0)$ spectrum obtained at the SEST. Financial support from the Swedish Science Research Council is gratefully acknowledged. FK was 
supported by APART (Austrian Programme for Advanced Research and Technology) from the Austrian Academy of Sciences and by the Austrian Science Fund Project P14365-PHY. FLS was supported by the Netherlands Organization for Scientific Research (NWO) grant 614.041 .004

\section{References}

Arndt, T. U., Fleischer, A. J., \& Sedlmayr, E. 1997, A\&A, 327, 614 Barthès, D., Luri, X., Alvarez, R., \& Mennessier, M. O. 1999, A\&AS, 140,55

Bergman, P., Kerschbaum, F., \& Olofsson, H. 2000, A\&A, 353, 257

Bernes, C. 1979, A\&A, 73, 67

Chandra, S., Maheshwari, V., \& Sharma, A. 1996, A\&AS, 117, 557

Crosas, M., \& Menten, K. M. 1997, ApJ, 483, 913

Doty, S. D., \& Leung, C. M. 1998, ApJ, 502, 898

Elitzur, M., \& Ivezic, Z. 2001, MNRAS, 327, 403

Flower, D. R. 2001, J. Phys B.: At. Mol. Opt. Phys., 34, 1

Flower, D. R., \& Launay, J. M. 1985, MNRAS, 214, 271

Glassgold, A. E., \& Huggins, P. J. 1983, MNRAS, 203, 517

Groenewegen, M. A. T. 1994, A\&A, 290, 531

Groenewegen, M. A. T., Baas, F., Blommaert, J. A. D. L., et al. 1999, A\&AS, 140, 197

Groenewegen, M. A. T., van der Veen, W. E. C. J., \& Matthews, H. E. 1998, A\&A, 338, 491

Habing, H. J. 1996, A\&AR, 7, 97

Habing, H. J., Tignon, J., \& Tielens, A. G. G. M. 1994, A\&A, 286, 523

Hale, D. D. S., Bester, M., Danchi, W. C., et al. 1997, ApJ, 490, 407

Höfner, S., \& Dorfi, E. A. 1997, A\&A, 319, 648

Huggins, P. J., \& Healy, A. P. 1986, ApJ, 304, 418

Huggins, P. J., Olofsson, H., \& Johansson, L. E. B. 1988, ApJ, 332, 1009

Jura, M., Chen, C., \& Plavchan, P. 2002, ApJ, in press

Jura, M., \& Kahane, C. 1999, ApJ, 521, 302

Kahane, C., Barnbaum, C., Uchida, K., Balm, S. P., \& Jura, M. 1998, ApJ, 500, 466

Kahane, C., \& Jura, M. 1994, A\&A, 290, 183

Kahane, C., \& Jura, M. 1996, A\&A, 310, 952

Kastner, J. H. 1992, ApJ, 401, 337

Kerschbaum, F. 1999, A\&A, 351, 627

Kerschbaum, F., \& Hron, J. 1996, A\&A, 308, 489

Kerschbaum, F., \& Olofsson, H. 1998, A\&A, 336, 654
Kerschbaum, F., \& Olofsson, H. 1999, A\&AS, 138, 299

Kerschbaum, F., Olofsson, H., \& Hron, J. 1996, A\&A, 311, 273

Kerschbaum, F., Olofsson, H., Hron, J., \& Lebzelter, T. 1997, Pulsating Stars - Recent Developments in Theory and Observation, 23rd meeting of the IAU, Joint Discussion 24, 26 August 1997, Kyoto, Japan, 24

Kholopov, P. N. 1990, General catalogue of variable stars, vol. 4, Reference tables, 4th edition, ed. P. N. Khopolov (Moscow: Nauka Publishing House)

Knapp, G. R., \& Morris, M. 1985, ApJ, 292, 640

Knapp, G. R., Young, K., Lee, E., \& Jorissen, A. 1998, ApJS, 117, 209

Kwan, J., \& Webster, Z. 1993, ApJ, 419, 674

Kwok, S. 1975, ApJ, 198, 583

Lindqvist, M., Olofsson, H., Lucas, R., et al. 1999, A\&A, 351, L1

Lindqvist, M., Olofsson, H., Winnberg, A., \& Nyman, L. A. 1992, A\&A, 263, 183

Mamon, G. A., Glassgold, A. E., \& Huggins, P. J. 1988, ApJ, 328, 797

Marengo, M., Ivezić, Ž., \& Knapp, G. R. 2001, MNRAS, 324, 1117

Mauron, N., \& Huggins, P. J. 2000, A\&A, 359, 707

Netzer, N., \& Knapp, G. R. 1987, ApJ, 323, 734

Neufeld, D. A., \& Kaufman, M. 1993, ApJ, 418, 263

Olofsson, H. 1999, in Asymptotic Giant Branch Stars, IAU Symp., 191,3

Olofsson, H., Bergman, P., Eriksson, K., \& Gustafsson, B. 1996, A\&A, 311, 587

Olofsson, H., Bergman, P., Lucas, R., et al. 2000, A\&A, 353, 583

Olofsson, H., Eriksson, K., Gustafsson, B., \& Carlstrom, U. 1993, ApJS, 87, 267

Ryde, N., \& Schöier, F. L. 2001, ApJ, 547, 384

Ryde, N., Schöier, F. L., \& Olofsson, H. 1999, A\&A, 345, 841

Schöier, F. L., \& Olofsson, H. 2000, A\&A, 359, 586

Schöier, F. L., \& Olofsson, H. 2001, A\&A, 368, 969

Schöier, F. L., Ryde, N., \& Olofsson, H. 2002, A\&A, in press

Schinke, R., Engel, V., Buck, U., Meyer, H., \& Diercksen, G. H. F. 1985, ApJ, 299, 939

van Langevelde, H. J., \& Spaans, M. 1993, MNRAS, 264, 597

van Zadelhoff, G.-J., Dullemond, C. P., Yates, J. A., et al. 2002, A\&A, submitted

Winters, J. M., Le Bertre, T., Jeong, K. S., Helling, C., \& Sedlmayr, E. 2000, A\&A, 361, 641

Young, K. 1995, ApJ, 445, 872 